\title{
On integral forms for vertex algebras associated with affine Lie algebras and lattices
}

\author{
Robert McRae
}

\begin{abstract}
We revisit the construction of integral forms for vertex (operator) algebras $V_{L}$ based on even lattices $L$ using generators instead of bases, and we construct integral forms for $V_{L}$-modules. We construct integral forms for vertex (operator) algebras based on highest-weight modules for affine Lie algebras and we exhibit natural generating sets. For vertex operator algebras in general, we give conditions showing when an integral form contains the standard conformal vector generating the Virasoro algebra. Finally, we study integral forms in contragredients of modules for vertex algebras.
\end{abstract}

\section{Introduction}

While vertex algebras are ordinarily assumed to be vector spaces over $\mathbb{C}$, or over any field of characteristic zero, the axioms, in particular the Jacobi identity, make sense for any commutative ring, and so it is natural to consider vertex algebras over $\mathbb{Z}$. In particular, it is natural to look for $\mathbb{Z}$-forms of vertex algebras over $\mathbb{C}$, by analogy with the construction of Lie algebras over $\mathbb{Z}$ using Chevalley bases. Given an integral form of a vertex algebra, one can then construct algebras over fields of prime characteristic $p$ by reducing structure constants $\bmod p$ and then extending scalars.

Integral forms for vertex algebras, and especially for vertex algebras constructed from even lattices, have been studied previously in $[\mathrm{B}],[\mathrm{P}]$, and $[\mathrm{DG}]$, and have been used in the modular moonshine program of Borcherds and Ryba $([\mathrm{R}],[\mathrm{BR} 1],[\mathrm{BR} 2, \mathrm{GL}])$. In this paper, we continue the study of integral forms for vertex algebras, revisiting some known results with new methods and proving new results as well. Our approach is based on finding generators for integral forms of vertex algebras. One advantage of this new method is that it allows the construction of an integral form containing desired elements without knowledge of the full structure in advance. This is particularly useful in constructing integral forms in vertex algebras based on affine Lie algebras and in constructing integral forms containing the standard conformal vector $\omega$ generating the Virasoro algebra. Further, defining an integral form $V_{\mathbb{Z}}$ for a vertex algebra $V$ to be the vertex subalgebra over $\mathbb{Z}$ generated by certain elements essentially reduces the problem of proving that $V_{\mathbb{Z}}$ is in fact an integral form of $V$ to the problem of showing that it is an integral form of $V$ as a vector space. In the case of vertex algebras based on lattices, this is easier than proving that an integral form of $V$ as a vector space is also a vertex subalgebra, which is the method of proof used in $[\mathrm{P}]$. 
In Section 2, we specify the classes of vertex algebras and modules that we will be studying and state some basic facts about vertex algebra integral forms. In Section 3, we construct integral forms for vertex algebras based on affine Lie algebras. In particular, we study affine Lie algebras based on a finite dimensional simple Lie algebra $\mathfrak{g}$ using the integral form of the universal enveloping algebra of an affine Lie algebra constructed in $[\mathrm{G}$ ] (see also [M] and $[\mathrm{P}]$ ). For these integral forms, we exhibit a natural set of generating elements. The recent paper GL] has constructed integral forms in level 1 affine Lie algebra vertex algebras using integral forms in lattice vertex algebras, but our results here can be applied to affine Lie algebra vertex algebras and modules of arbitrary integral level.

In Section 4, we study integral forms for vertex algebras $V_{L}$ based on even lattices $L$. In his research announcement [B], Borcherds defined an integral form for such a vertex algebra and exhibited a $\mathbb{Z}$-basis for this form. It has been proved in $[\mathrm{P}]$ and $[\mathrm{DG}]$ that this structure is in fact an integral form, essentially by showing that the vertex algebra product of any two members of Borcherds' $\mathbb{Z}$-basis is a $\mathbb{Z}$-linear combination of basis elements. In Section 4, we provide an alternate proof by defining the integral form to be the vertex subalgebra over $\mathbb{Z}$ generated by a natural generating set and then proving that the resulting structure is an integral form of the vector space $V_{L}$. We also show that our definition of the integral form is equivalent to the definition in [B]. Further, we construct integral forms in modules for lattice vertex algebras. This problem is slightly more subtle than the problem of constructing an integral form in the algebra due to the nature of a central extension of the dual lattice $L^{\circ}$ that is needed to construct modules for $V_{L}$.

In Section 5, we consider when an integral form for a vertex operator algebra contains the standard conformal element $\omega$ generating the Virasoro algebra. For a vertex algebra based on a lattice $L$ we solve this problem by proving that the integral form constructed in Section 4 contains $\omega$ if and only if $L$ is self-dual (the "if" direction was observed in [BR1]). More generally, we give conditions under which an integral form of a vertex operator algebra can be extended to include a multiple of $\omega$. Finally, in Section 6, we consider the construction of integral forms in contragredient modules, applying this to the situation in which the vertex algebra has a non-degenerate invariant bilinear form. Some of these results have already appeared in [DG] in the context of invariant forms on vertex algebras, but we formulate them more generally here in the context of contragredient modules.

Acknowledgements This paper is part of my thesis work at Rutgers University; I am very grateful to my advisor James Lepowsky for many helpful discussions and encouragement. This research was partially supported by NSF grant DMS-0701176.

\section{Vertex algebras and integral forms}

In this paper, by vertex algebra we will mean the structure as defined by Borcherds in [B] (see also [LL for an equivalent definition and proof of the equivalence). We use the definition of [FLM] for vertex operator algebra (see also [LL]); that is, a vertex operator algebra is a vertex algebra with a conformal vector $\omega$ satisfying the usual properties. We can weaken the notion of vertex operator algebra by dropping the grading restriction conditions on the $L(0)$ weight spaces: that is, we allow infinitely many (non-zero) spaces of negative weight and we 
allow weight spaces to be infinite dimensional. Such a structure is called a conformal vertex algebra in [HLZ]. In this paper, we will use the definition of module for a vertex (operator) algebra or conformal vertex algebra as in [LL and [HLZ]. In particular, if a module has a weight grading, the weights are allowed to be in $\mathbb{C}$.

The specific conformal vertex algebras and their modules that we will study in this paper have an additional grading by an abelian group, in addition to the weight grading. Thus we need the notion of strongly $A$-graded conformal vertex algebra, $A$ an abelian group, as formulated in [HLZ): a conformal vertex algebra $V$ is strongly $A$-graded if there is an additional grading by $A$,

$$
V=\coprod_{\alpha \in A} V^{\alpha},
$$

compatible with the weight grading in the sense that for any $\alpha \in A$,

$$
V^{\alpha}=\coprod_{n \in \mathbb{Z}} V_{n}^{\alpha},
$$

where $V_{n}^{\alpha}=V^{\alpha} \cap V_{n}$ for $n \in \mathbb{Z}$. Further, we require that $\mathbf{1} \in V_{0}^{0}, \omega \in V_{2}^{0}$, and if $v \in V^{\alpha}$, then

$$
Y(v, x) V^{\beta} \subseteq V^{\alpha+\beta}\left[\left[x, x^{-1}\right]\right] .
$$

Finally, we want the grading restriction conditions: for any fixed $\alpha \in A, V_{n}^{\alpha}=0$ for $n$ sufficiently negative and $\operatorname{dim} V_{n}^{\alpha}<\infty$ for any $n$. A module for a strongly $A$-graded conformal vertex algebra $V$ is a module $W$ for $V$ as conformal vertex algebra with an additional $B$ grading, $B$ an abelian group containing $A$. The $B$-grading of $W$ and the $\mathbb{C}$-grading by conformal weight are compatible, the grading restriction conditions hold, and $V^{\alpha}$ maps $W^{\beta}$ into $W^{\alpha+\beta}$, as above.

The notion of vertex algebra over $\mathbb{Z}$ makes sense because all numerical coefficients in the formal delta functions appearing in the Jacobi identity [FLM] are integers. For convenience, we will call vertex algebras over $\mathbb{Z}$ vertex rings in this paper, and we will call $\mathbb{Z}$-subalgebras of vertex algebras vertex subrings. Recall that an integral form of a vector space $V$ is a free abelian group $V_{\mathbb{Z}}$ such that the canonical map

$$
\mathbb{C} \otimes_{\mathbb{Z}} V_{\mathbb{Z}} \rightarrow V
$$

given by

$$
c \otimes_{\mathbb{Z}} v \mapsto c v
$$

for $c \in \mathbb{C}$ and $v \in V$, is an isomorphism. That is, $V_{\mathbb{Z}}$ is the $\mathbb{Z}$-span of a basis of $V$. Thus given a vertex algebra $V$ over $\mathbb{C}$ (or any field of characteristic zero), we can define an integral form of $V$ to be a vertex subring $V_{\mathbb{Z}} \subseteq V$ which is an integral form of the vector space $V$. In other words, $V_{\mathbb{Z}}$ is the $\mathbb{Z}$-span of a basis for $V$, it contains the vacuum vector $\mathbf{1}$, and it is closed under vertex algebra products. The notion of module for a vertex algebra over $\mathbb{Z}$ also makes sense. Thus if $V$ is a vertex algebra over $\mathbb{C}$ having an integral form $V_{\mathbb{Z}}$ and $W$ is a $V$-module, we can define an integral form for $W$ to be a $V_{\mathbb{Z}}$-submodule $W_{\mathbb{Z}} \subseteq W$ which is an integral form of the vector space $W$. That is, $W_{\mathbb{Z}}$ is the $\mathbb{Z}$-span of a basis for $W$, and it is preserved by vertex operators from $V_{\mathbb{Z}}$. 
If $V$ is also a vertex operator algebra or conformal vertex algebra, and so has a conformal element $\omega$, we do not require an integral form of $V$ to contain $\omega$. Such a requirement would disallow many interesting integral forms. However, in this paper, we will require an integral form $V_{\mathbb{Z}}$ of $V$ to be compatible with the weight grading:

$$
V_{\mathbb{Z}}=\coprod_{n \in \mathbb{Z}} V_{n} \cap V_{\mathbb{Z}}
$$

where $V_{n}$ is the weight space with $L(0)$-eigenvalue $n$. Moreover, if $V$ is a strongly $A$-graded conformal vertex algebra, we require $V_{\mathbb{Z}}$ to be compatible with the $A \times \mathbb{C}$-gradation:

$$
V_{\mathbb{Z}}=\coprod_{\alpha \in A, n \in \mathbb{Z}} V_{n}^{\alpha} \cap V_{\mathbb{Z}}
$$

We require the analogues of these compatibility conditions for modules for a conformal vertex algebra or strongly $A$-graded conformal vertex algebra. One could assume different requirements on the relation of $\omega$ to $V_{\mathbb{Z}}$; for instance, in [DG] it is required that some integer multiple of $\omega$ be in $V_{\mathbb{Z}}$.

We conclude this section with the following useful general results on vertex rings:

Proposition 2.1. Suppose $V$ is a vertex algebra; for a subset $S$ of $V$, denote by $\langle S\rangle_{\mathbb{Z}}$ the vertex subring generated by $S$. Then $\langle S\rangle_{\mathbb{Z}}$ is the $\mathbb{Z}$-span of coefficients of products of the form

$$
Y\left(u_{1}, x_{1}\right) \ldots Y\left(u_{k}, x_{k}\right) \mathbf{1}
$$

where $u_{1}, \ldots u_{k} \in S$. Moreover, if $W$ is a $V$-module, the $\langle S\rangle_{\mathbb{Z}}$-submodule generated by a subset $T$ of $W$ is the $\mathbb{Z}$-span of coefficients of products of the form

$$
Y\left(u_{1}, x_{1}\right) \ldots Y\left(u_{k}, x_{k}\right) w
$$

where $u_{1}, \ldots u_{k} \in S$ and $w \in T$.

Proof. Use the proof of Proposition 3.9.3 in [LL], noting that all numerical coefficients in the Jacobi identity are integers.

Remark 2.2. Proposition 2.1 applies even if $\langle S\rangle_{\mathbb{Z}}$ and the $\langle S\rangle_{\mathbb{Z}}$-submodule generated by $T$ are not integral forms of their respective vector spaces.

Proposition 2.3. Suppose $V$ is a vertex algebra with integral form $V_{\mathbb{Z}}$. Then $V_{\mathbb{Z}} \cap \mathbb{C} \mathbf{1}=\mathbb{Z} \mathbf{1}$.

Proof. Since $\mathbf{1} \in V_{\mathbb{Z}}$, it is clear that $\mathbb{Z} \mathbf{1} \subseteq V_{\mathbb{Z}} \cap \mathbb{C} \mathbf{1}$. On the other hand, since $V_{\mathbb{Z}}$ is the $\mathbb{Z}$-span of a basis $\left\{v_{i}\right\}$ for $V, \mathbf{1}=\sum_{i} n_{i} v_{i}$ where $n_{i} \in \mathbb{Z}$. If $c \mathbf{1}=\sum_{i} c n_{i} v_{i} \in V_{\mathbb{Z}} \cap \mathbb{C} \mathbf{1}$ for $c \in \mathbb{C}$, then $c n_{i} \in \mathbb{Z}$ for each $i$, so $c \in \mathbb{Q}$. If $c \notin \mathbb{Z}$, by subtracting off an integer multiple of $\mathbf{1}$ from $c \mathbf{1}$, we may assume $0<c<1$. Since $V_{\mathbb{Z}}$ is closed under vertex operators and $Y(\mathbf{1}, x)=1_{V}$, we see that $c^{n} \mathbf{1} \in V_{\mathbb{Z}}$ for all $n \geq 0$, contradicting the assumption that $V_{\mathbb{Z}}$ is an integral form of $V$. Thus $c \in \mathbb{Z}$. 


\section{Integral forms in vertex algebras based on affine Lie algebras}

We now recall the construction of vertex algebras based on affine Lie algebras (see [FZ] and [LL for more details). Suppose that $\mathfrak{g}$ is a finite dimensional Lie algebra, with symmetric invariant form $\langle\cdot, \cdot\rangle$. Then we can form the affine Lie algebra

$$
\widehat{\mathfrak{g}}=\mathfrak{g} \otimes \mathbb{C}\left[t, t^{-1}\right] \oplus \mathbb{C} \mathbf{k}
$$

where $\mathbf{k}$ is central and all other brackets are determined by

$$
\left[a \otimes t^{m}, b \otimes t^{n}\right]=[a, b] \otimes t^{m+n}+m\langle a, b\rangle \delta_{m+n, 0} \mathbf{k},
$$

where $a, b \in \mathfrak{g}$ and $m, n \in \mathbb{Z}$. The affine Lie algebra has the decomposition

$$
\widehat{\mathfrak{g}}=\widehat{\mathfrak{g}}_{+} \oplus \widehat{\mathfrak{g}}_{0} \oplus \widehat{\mathfrak{g}}_{-}
$$

where

$$
\widehat{\mathfrak{g}}_{ \pm}=\coprod_{n \in \pm \mathbb{Z}_{+}} \mathfrak{g} \otimes t^{n}, \quad \widehat{\mathfrak{g}}_{0}=\mathfrak{g} \otimes t^{0} \oplus \mathbb{C} \mathbf{k}
$$

If $U$ is a finite dimensional $\mathfrak{g}$-module, then $U$ becomes a $\widehat{\mathfrak{g}}_{+} \oplus \widehat{\mathfrak{g}}_{0}$-module on which $\widehat{\mathfrak{g}}_{+}$acts trivially, $\mathfrak{g} \otimes t^{0}$ acts as $\mathfrak{g}$, and $\mathbf{k}$ acts as some scalar $\ell \in \mathbb{C}$. Then we can form the generalized Verma module

$$
V_{\widehat{\mathfrak{g}}}(\ell, U)=U(\widehat{\mathfrak{g}}) \otimes_{U\left(\widehat{\mathfrak{g}}_{+} \oplus \widehat{\mathfrak{g}}_{0}\right)} U
$$

which is a $\widehat{\mathfrak{g}}$-module of level $\ell$; it has a unique maximal submodule and thus a unique irreducible quotient, $L_{\widehat{\mathfrak{g}}}(\ell, U)$. We use $V_{\widehat{\mathfrak{g}}}(\ell, 0)$ to denote $V_{\widehat{\mathfrak{g}}}(\ell, U)$ where $U$ is the trivial $\mathfrak{g}$ module, and $L_{\widehat{\mathfrak{g}}}(\ell, 0)$ to denote its irreducible quotient. If we write the trivial $\mathfrak{g}$-module with $\mathbf{k}$ acting as $\ell$ as $\mathbb{C} 1_{\ell}$, and then write $\mathbf{1}=1 \otimes 1_{\ell} \in V_{\widehat{\mathfrak{g}}}(\ell, 0)$, then we can write

$$
V_{\widehat{\mathfrak{g}}}(\ell, 0)=U\left(\widehat{\mathfrak{g}}_{-}\right) \mathbf{1}
$$

so $V_{\widehat{\mathfrak{g}}}(\ell, 0)$ is spanned by elements of the form

$$
a_{1}\left(-n_{1}\right) \ldots a_{k}\left(-n_{k}\right) \mathbf{1}
$$

where $a_{i} \in \mathfrak{g}, n_{i} \in \mathbb{Z}_{+}$, and we use the notation $a(n)$ to denote the action of $a \otimes t^{n}$ on $\widehat{\mathfrak{g}}$-modules.

The generalized Verma module $V_{\widehat{\mathfrak{g}}}(\ell, 0)$ is a vertex algebra, as are all its quotients, including $L_{\widehat{\mathfrak{g}}}(\ell, 0)$, which is a simple vertex algebra. The vertex algebra structure is determined by

$$
Y(a(-1) \mathbf{1}, x)=\sum_{n \in \mathbb{Z}} a(n) x^{-n-1} .
$$

(The elements $a(-1) \mathbf{1}$ generate $V_{\widehat{\mathfrak{g}}}(\ell, 0)$, and thus also its quotients, as vertex algebras.) As long as $\ell \neq-h$, where $h$ is the dual Coxeter number of $\mathfrak{g}, V_{\widehat{\mathfrak{g}}}(\ell, 0)$ is also a vertex operator algebra, with conformal vector given by

$$
\omega=\frac{1}{2(\ell+h)} \sum_{i=1}^{\operatorname{dim} \mathfrak{g}} u_{i}(-1) u_{i}^{\prime}(-1) \mathbf{1}
$$


where $\left\{u_{i}\right\}$ is any basis of $\mathfrak{g}$ and $\left\{u_{i}^{\prime}\right\}$ is the corresponding dual basis with respect to the form $\langle\cdot, \cdot\rangle$. The generalized Verma modules $V_{\widehat{\mathfrak{g}}}(\ell, U)$ for finite-dimensional $\mathfrak{g}$-modules $U$ are $V_{\widehat{\mathfrak{g}}}(\ell, 0)$-modules, as well as all quotients of $V_{\widehat{\mathfrak{g}}}(\ell, U)$; the irreducible $V_{\widehat{\mathfrak{g}}}(\ell, 0)$-modules consist precisely of the $\widehat{\mathfrak{g}}$-modules $L_{\widehat{\mathfrak{g}}}(\ell, U)$ where $U$ is a finite-dimensional irreducible $\mathfrak{g}$-module.

In order to construct integral forms in these vertex algebras and modules, we will assume that $\mathfrak{g}$ has an integral form. That is, we assume that $\mathfrak{g}$ has a basis whose $\mathbb{Z}$-span $\mathfrak{g}_{\mathbb{Z}}$ is closed under the bracket and such that the form $\langle\cdot, \cdot\rangle$ is integer-valued on $\mathfrak{g}_{\mathbb{Z}}$. Then $\widehat{\mathfrak{g}}$ also has the integral form

$$
\widehat{\mathfrak{g}}_{\mathbb{Z}}=\mathfrak{g}_{\mathbb{Z}} \otimes_{\mathbb{Z}} \mathbb{Z}\left[t, t^{-1}\right] \oplus \mathbb{Z} \mathbf{k}
$$

For example, if $\mathfrak{h}$ is a finite-dimensional abelian Lie algebra with a symmetric non-degenerate form, any full-rank integral lattice $L \subseteq \mathfrak{h}$ is an integral form of $\mathfrak{h}$. If $\mathfrak{g}$ is a finite-dimensional simple Lie algebra, we can take for $\mathfrak{g}_{\mathbb{Z}}$ the $\mathbb{Z}$-span of a Chevalley basis of $\mathfrak{g}$. (There do exist symmetric invariant non-degenerate integer-valued bilinear forms on such a $\mathfrak{g}_{\mathbb{Z}}$ because the Killing form $\kappa(a, b)=\operatorname{Tr}(\operatorname{ad} a \operatorname{ad} b)$ is integral on $\left.\mathfrak{g}_{\mathbb{Z}} \cdot\right)$

Now suppose that $U_{\mathbb{Z}}(\widehat{\mathfrak{g}})$ is an integral form of the universal enveloping algebra $U(\widehat{\mathfrak{g}})$ such that

$$
U_{\mathbb{Z}}(\widehat{\mathfrak{g}})=U_{\mathbb{Z}}\left(\widehat{\mathfrak{g}}_{-}\right) U_{\mathbb{Z}}\left(\widehat{\mathfrak{g}}_{0}\right) U_{\mathbb{Z}}\left(\widehat{\mathfrak{g}}_{+}\right),
$$

where $U_{\mathbb{Z}}\left(\widehat{\mathfrak{g}}_{ \pm}\right)$and $U_{\mathbb{Z}}\left(\widehat{\mathfrak{g}}_{0}\right)$ are integral forms of $U\left(\widehat{\mathfrak{g}}_{ \pm}\right)$and $U\left(\widehat{\mathfrak{g}}_{0}\right)$, respectively. We also assume that $U_{\mathbb{Z}}\left(\widehat{\mathfrak{g}}_{ \pm}\right)$are graded in the sense that

$$
U_{\mathbb{Z}}\left(\widehat{\mathfrak{g}}_{ \pm}\right)=\coprod_{n \in \mathbb{Z}} U_{\mathbb{Z}}\left(\widehat{\mathfrak{g}}_{ \pm}\right) \cap U\left(\widehat{\mathfrak{g}}_{ \pm}\right)_{n}
$$

where $U\left(\widehat{\mathfrak{g}}_{ \pm}\right)_{n}$ is the space spanned by monomials of the form

$$
\left(g_{1} \otimes t^{n_{1}}\right) \cdots\left(g_{k} \otimes t^{n_{k}}\right)
$$

where $g_{1}, \ldots, g_{k} \in \mathfrak{g}$ and $n=n_{1}+\ldots+n_{k}$. We want to use such a $U_{\mathbb{Z}}(\widehat{\mathfrak{g}})$ to obtain integral forms in generalized Verma modules $V_{\widehat{\mathfrak{g}}}(\ell, U)$ and their irreducible quotients when $\ell \in \mathbb{Z}$.

Remark 3.1. Perhaps the simplest way to obtain such a $U_{\mathbb{Z}}(\widehat{\mathfrak{g}})$ is to take a $\mathbb{Z}$-basis $\left\{u_{i}\right\}$ of $\mathfrak{g}_{\mathbb{Z}}$ and extend it to a $\mathbb{Z}$-basis $\left\{u_{i} \otimes t^{n}, \mathbf{k}\right\}$ of $\widehat{\mathfrak{g}}_{\mathbb{Z}}$. Then $U_{\mathbb{Z}}(\widehat{\mathfrak{g}})$ may be defined as the $\mathbb{Z}$-span of ordered monomials in these basis elements, ordered in such a way that the decomposition (3.11) holds. This is certainly an integral form of $U(\widehat{\mathfrak{g}})$ as a vector space, and in fact it is an integral form of $U(\widehat{\mathfrak{g}})$ as an associative algebra since it includes 1 and since a product of two ordered monomials is an integral combination of ordered monomials: this is because in any unordered monomial, the order of any two basis elements of $\widehat{\mathfrak{g}}_{\mathbb{Z}}$ can be switched at the cost of a commutator term which is an integral linear combination of monomials of lower degree.

By an integral form $U_{\mathbb{Z}}$ of a $\widehat{\mathfrak{g}}_{0}$-module $U$, we will mean an integral form of $U$ as a vector space which is invariant under $U_{\mathbb{Z}}\left(\widehat{\mathfrak{g}}_{0}\right)$, where $U_{\mathbb{Z}}\left(\widehat{\mathfrak{g}}_{0}\right)$ is as in (3.11). For example, if $U_{\mathbb{Z}}(\widehat{\mathfrak{g}})$ is constructed as in Remark 3.1 and $U$ as a vector space has an integral form $U_{\mathbb{Z}}$ which is a $\mathfrak{g}_{\mathbb{Z}}$-module, and $\mathbf{k}$ acts on $U$ as an integer, then $U_{\mathbb{Z}}$ is an integral form of $U$.

Theorem 3.2. Suppose $U_{\mathbb{Z}}$ is an integral form of a finite-dimensional $\widehat{\mathfrak{g}}_{0}$-module $U$ on which $\mathbf{k}$ acts as $\ell$, and suppose $W$ is $V_{\widehat{\mathfrak{g}}}(\ell, U)$ or its irreducible quotient $L_{\widehat{\mathfrak{g}}}(\ell, U)$. Then $W_{\mathbb{Z}}=$ $U_{\mathbb{Z}}(\widehat{\mathfrak{g}}) U_{\mathbb{Z}}$ is an integral form of $W$ as a vector space, and $W_{\mathbb{Z}}$ is compatible with the conformal weight grading of $W$. Moreover, $W_{\mathbb{Z}}$ is invariant under $U_{\mathbb{Z}}(\widehat{\mathfrak{g}})$. 
Proof. By definition, $W_{\mathbb{Z}}$ is invariant under $U_{\mathbb{Z}}(\widehat{\mathfrak{g}})$. Also, since $\widehat{\mathfrak{g}}_{+} \cdot U=0$ and since $U_{\mathbb{Z}}\left(\widehat{\mathfrak{g}}_{0}\right) U_{\mathbb{Z}} \subseteq U_{\mathbb{Z}}$

$$
U_{\mathbb{Z}}(\widehat{\mathfrak{g}}) U_{\mathbb{Z}}=U_{\mathbb{Z}}\left(\widehat{\mathfrak{g}}_{-}\right) U_{\mathbb{Z}}\left(\widehat{\mathfrak{g}}_{0}\right) U_{\mathbb{Z}}\left(\widehat{\mathfrak{g}}_{+}\right) U_{\mathbb{Z}}=U_{\mathbb{Z}}\left(\widehat{\mathfrak{g}}_{-}\right) U_{\mathbb{Z}}
$$

Since we assume $U_{\mathbb{Z}}\left(\widehat{\mathfrak{g}}_{-}\right)$is graded as in (3.12), $W_{\mathbb{Z}}$ is graded by conformal weight and the intersection of $W_{\mathbb{Z}}$ with each weight space is spanned by finitely many vectors. In fact, an upper bound on the number of vectors required to span the weight space of weight $n$ higher than the lowest weight space is $\operatorname{dim} U\left(\widehat{\mathfrak{g}}_{-}\right)_{n} \cdot \operatorname{dim} U<\infty$. Moreover, since $W=U\left(\widehat{\mathfrak{g}}_{-}\right) U$, it follows that $W_{\mathbb{Z}}$ spans $W$ as a vector space. To prove the theorem, that is, to prove that $W$ is linearly isomorphic to $\mathbb{C} \otimes_{\mathbb{Z}} W_{\mathbb{Z}}$, we need to show that if a set of vectors in $W_{\mathbb{Z}}$ is linearly independent over $\mathbb{Z}$, then they are also linearly independent over $\mathbb{C}$.

In the case that $W=V_{\widehat{\mathfrak{g}}}(\ell, U)$, then $W_{\mathbb{Z}}$ is isomorphic as a $\mathbb{Z}$-module to $U_{\mathbb{Z}}\left(\widehat{\mathfrak{g}}_{-}\right) \otimes_{\mathbb{Z}} U_{\mathbb{Z}}$, which is a free graded $\mathbb{Z}$-module whose weight spaces have rank equal to the dimension of the weight spaces of $W \cong U\left(\widehat{\mathfrak{g}}_{-}\right) \otimes_{\mathbb{C}} U$ since $U_{\mathbb{Z}}\left(\widehat{\mathfrak{g}}_{-}\right)$is a graded integral form of $U\left(\widehat{\mathfrak{g}}_{-}\right)$. This proves the theorem in this case.

In the case that $W=L_{\widehat{\mathfrak{g}}}(\ell, U)$, we use a slight generalization of the proof of Theorem 11.3 in [G], which is an analogue of Lemma 12 in [S]; see also Theorem 27.1 in [H]. We first observe that $W_{\mathbb{Z}} \cap U=U_{\mathbb{Z}}$, and we use $\left\{u_{i}\right\}$ to denote a $\mathbb{Z}$-base of $U_{\mathbb{Z}}$. Next, we observe that if $w \in W_{\mathbb{Z}}$ is non-zero, there is some $y \in U_{\mathbb{Z}}\left(\widehat{\mathfrak{g}}_{+}\right)$such that the component of $y \cdot w$ in $U$ is non-zero; this is because otherwise $w$ would generate a proper $\widehat{\mathfrak{g}}$-submodule in $L_{\widehat{\mathfrak{g}}}(\ell, U)$, which is impossible. Note that the component of $y \cdot w$ in $U$ is in $U_{\mathbb{Z}}$ since $W_{\mathbb{Z}}$ is graded and invariant under $U_{\mathbb{Z}}(\widehat{\mathfrak{g}})$.

Now we prove that $W_{\mathbb{Z}}$ is an integral form of $W$ by contradiction. Suppose that $\left\{w_{i}\right\}_{i=1}^{k}$ is a minimal set contained in $W_{\mathbb{Z}}$ that is linearly independent over $\mathbb{Z}$ but not over $\mathbb{C}$; note that for this to happen, $k \geq 2$. Then

$$
\sum_{i=1}^{k} c_{i} w_{i}=0
$$

for $c_{i} \in \mathbb{C}^{\times}$. Take $y \in U\left(\widehat{\mathfrak{g}}_{+}\right)$such that the component $\left(y \cdot w_{1}\right)_{U}$ of $y \cdot w_{1}$ in $U_{\mathbb{Z}}$ is non-zero. In particular, for some basis element $u_{j}$ of $U_{\mathbb{Z}}$, there is a non-zero integer $m_{1}$ such that the projection of $\left(y \cdot w_{1}\right)_{U}$ to $\mathbb{Z} u_{j}$ (with respect to the basis $\left\{u_{i}\right\}$ ) is $m_{1} u_{j}$. Also, for $2 \leq i \leq k$, the projection of $\left(y \cdot w_{i}\right)_{U}$ to $\mathbb{Z} u_{j}$ is $m_{i} u_{j}$ for some integer $m_{i}$. Thus, since

$$
0=y \cdot \sum_{i=1}^{k} c_{i} w_{i}=\sum_{i=1}^{k} c_{i}\left(y \cdot w_{i}\right)
$$

we obtain

$$
\sum_{i=1}^{k} c_{i} m_{i}=0
$$

because $\left\{u_{i}\right\}$ forms a basis for $U$ over $\mathbb{C}$ as well as a basis for $U_{\mathbb{Z}}$ over $\mathbb{Z}$. Thus

$$
0=m_{1}\left(\sum_{i=1}^{k} c_{i} w_{i}\right)-\left(\sum_{i=1}^{k} c_{i} m_{i}\right) w_{1}=\sum_{i=1}^{k}\left(m_{1} c_{i} w_{i}-m_{i} c_{i} w_{1}\right)=\sum_{i=2}^{k} c_{i}\left(m_{1} w_{i}-m_{i} w_{1}\right)
$$

Since $m_{1} \neq 0$, the vectors $m_{1} w_{i}-m_{i} w_{1}$ for $2 \leq i \leq k$ are thus in $W_{\mathbb{Z}}$, linearly independent over $\mathbb{Z}$, and linearly dependent over $\mathbb{C}$, which contradicts the minimality of $\left\{w_{i}\right\}_{i=1}^{k}$. 
We next prove a general result on vertex algebraic integral forms that applies to any finite-dimensional Lie algebra $\mathfrak{g}$ having an integral form.

Proposition 3.3. Suppose $\mathfrak{g}_{\mathbb{Z}}$ is an integral form of $\mathfrak{g}, U_{\mathbb{Z}}(\widehat{\mathfrak{g}})$ is constructed as in Remark 3.1, and $\ell \in \mathbb{Z}$. Then the integral form $V_{\widehat{\mathfrak{g}}}(\ell, 0)_{\mathbb{Z}}$ given by Theorem 3.2 $i$ s the vertex subring generated by the vectors $a(-1) \mathbf{1}$ for $a \in \mathfrak{g}_{\mathbb{Z}}$. Moreover, if $U$ is a finite-dimensional $\mathfrak{g}$-module with integral form $U_{\mathbb{Z}}, V_{\widehat{\mathfrak{g}}}(\ell, U)_{\mathbb{Z}}$ and $L_{\mathfrak{\mathfrak { g }}}(\ell, U)_{\mathbb{Z}}$ are the $V_{\widehat{\mathfrak{g}}}(\ell, 0)_{\mathbb{Z}}$-modules generated by $U_{\mathbb{Z}}$.

Proof. Let $W$ be the module $V_{\widehat{\mathfrak{g}}}(\ell, U)$ or $L_{\widehat{\mathfrak{g}}}(\ell, U)$, where $U$ is possibly $\mathbb{C} 1_{\ell}$. From the construction in Remark 3.1 ,

$$
W_{\mathbb{Z}}=U_{\mathbb{Z}}\left(\widehat{\mathfrak{g}}_{-}\right) U_{\mathbb{Z}}
$$

is the integral span of vectors of the form

$$
a_{1}\left(-n_{1}\right) \cdots a_{k}\left(-n_{k}\right) u
$$

where $a_{i} \in \mathfrak{g}_{\mathbb{Z}}, n_{i}>0$, and $u \in U_{\mathbb{Z}}$. On the other hand, since

$$
Y(a(-1) \mathbf{1}, x)=\sum_{n \in \mathbb{Z}} a(n) x^{-n-1}
$$

for $a \in \mathfrak{g}$, Proposition 2.1 implies that the vertex subring generated by the $a(-1) \mathbf{1}$ is the integral span of vectors of the form

$$
a_{1}\left(n_{1}\right) \cdots a_{k}\left(n_{k}\right) \mathbf{1}
$$

for $a_{i} \in \mathfrak{g}_{\mathbb{Z}}$ and $n_{i} \in \mathbb{Z}$. In the case that $U=\mathbb{C}_{\ell}$, this is the same as (3.20) because $a(n) \mathbf{1}=0$ if $n>0$ and any $a_{i}\left(n_{i}\right)$ occurring in (3.22) with $n_{i}>0$ can be moved to the right using the commutation relations (3.2). This proves the first assertion of the proposition, and the second follows similarly.

Corollary 3.4. In the setting of Proposition [3.3, $L_{\widehat{\mathfrak{g}}}(\ell, 0)_{\mathbb{Z}}$ is the vertex ring generated by vectors of the form $a(-1) \mathbf{1}$ for $a \in \mathfrak{g}_{\mathbb{Z}}$.

Proof. This follows because $L_{\widehat{\mathfrak{g}}}(\ell, 0)_{\mathbb{Z}}$ is the integral span of vectors of the form (3.22), which is also the vertex subring generated by vectors of the form $a(-1) \mathbf{1}$ for $a \in \mathfrak{g}_{\mathbb{Z}}$, just as in the proof of Proposition 3.3 .

If $\mathfrak{g}$ is a finite-dimensional simple Lie algebra, there is another way to obtain integral structure in modules for $\widehat{\mathfrak{g}}(\mathrm{G}]$; see also $[\mathrm{M}],[\mathrm{P}])$. Consider a Chevalley basis for $\mathfrak{g}$ where for a root $\alpha, x_{\alpha}$ is the basis vector of the corresponding root space. Then the subring $U_{\mathbb{Z}}(\widehat{\mathfrak{g}}) \subseteq U(\widehat{\mathfrak{g}})$ generated by the elements $\left(x_{\alpha} \otimes t^{n}\right)^{k} / k$ ! for $k \geq 0, n \in \mathbb{Z}$, and $\alpha$ a root of $\mathfrak{g}$ is an integral form of $U(\widehat{\mathfrak{g}})$ which satisfies (3.11) and (3.12); moreover, if $U$ is a finitedimensional irreducible $\mathfrak{g}$-module with highest weight vector $v_{0}$, then $U_{\mathbb{Z}}\left(\widehat{\mathfrak{g}}_{0}\right) \cdot v_{0}$ is an integral form of $U$ as long as $\mathbf{k}$ acts as an integer $\ell$; here $U_{\mathbb{Z}}\left(\widehat{\mathfrak{g}}_{0}\right)=U_{\mathbb{Z}}(\widehat{\mathfrak{g}}) \cap U\left(\widehat{\mathfrak{g}}_{0}\right)$. Consequently any finite-dimensional $\mathfrak{g}$-module has a $U_{\mathbb{Z}}\left(\widehat{\mathfrak{g}}_{0}\right)$-invariant integral form since finite-dimensional $\mathfrak{g}$-modules are completely reducible. Thus we can apply Theorem 3.2 and conclude that for any finite-dimensional $\mathfrak{g}$-module $U$, the $\widehat{\mathfrak{g}}$-modules $V_{\widehat{\mathfrak{g}}}(\ell, U)$ and $L_{\widehat{\mathfrak{g}}}(\ell, U)$ when $\ell \in \mathbb{Z}$ have $U_{\mathbb{Z}}(\widehat{\mathfrak{g}})$-invariant integral forms which are compatible with the conformal weight gradings.

We can now show that the integral forms obtained using this $U_{\mathbb{Z}}(\widehat{\mathfrak{g}})$ for a finite-dimensional simple Lie algebra $\mathfrak{g}$ have vertex algebraic integral structure: 
Theorem 3.5. Suppose $\ell \in \mathbb{Z}$; the integral form $V_{\widehat{\mathfrak{g}}}(\ell, 0)_{\mathbb{Z}}$ is the vertex subring of $V_{\widehat{\mathfrak{g}}}(\ell, 0)$ generated by the vectors $\frac{x_{\alpha}(-1)^{k}}{k !} \mathbf{1}$ where $k \geq 0$ and $x_{\alpha}$ is the root vector corresponding to the root $\alpha$ in the chosen Chevalley basis of $\mathfrak{g}$. Moreover, if $U$ is a finite-dimensional $\mathfrak{g}$ module with integral form $U_{\mathbb{Z}}$ and $W$ is $V_{\widehat{\mathfrak{g}}}(\ell, U)$ or $L_{\mathfrak{\mathfrak { g }}}(\ell, U)$, then $W_{\mathbb{Z}}$ is the $V_{\widehat{\mathfrak{g}}}(\ell, 0)_{\mathbb{Z}}$-module generated by $U_{\mathbb{Z}}$.

Proof. Since $U_{\mathbb{Z}}(\widehat{\mathfrak{g}})$ is generated as a ring by the divided powers $\left(x_{\alpha} \otimes t^{n}\right)^{k} / k$ ! where $\alpha$ is a root of $\mathfrak{g}$ and $k \geq 0$, we can express $W_{\mathbb{Z}}=U_{\mathbb{Z}}(\widehat{\mathfrak{g}}) \cdot U_{\mathbb{Z}}$ as the $\mathbb{Z}$-span of products of the form

$$
\frac{x_{\alpha_{1}}\left(m_{1}\right)^{k_{1}}}{k_{1} !} \cdots \frac{x_{\alpha_{n}}\left(m_{n}\right)^{k_{n}}}{k_{n} !} \cdot u
$$

where the $\alpha_{i}$ are roots of $\mathfrak{g}, m_{i} \in \mathbb{Z}, k_{i} \geq 0$, and $u \in U_{\mathbb{Z}}$ (where $U$ could be $\mathbb{C} 1_{\ell}$, in which case $u=1$ ). By Proposition 2.1, we need to show that the $\mathbb{Z}$-span of such products equals the $\mathbb{Z}$-span of coefficients of products of the form

$$
Y\left(\frac{x_{\alpha_{1}}(-1)^{k_{1}}}{k_{1} !}, x_{1}\right) \cdots Y\left(\frac{x_{\alpha_{n}}(-1)^{k_{n}}}{k_{n} !}, x_{n}\right) u
$$

First we will analyze the vertex operator associated to a generator $x_{\alpha}(-1)^{k} / k$ !, where $\alpha$ is any root of $\mathfrak{g}$. First, observe that for any $m, n \in \mathbb{Z}$,

$$
\left[x_{\alpha}(m), x_{\alpha}(n)\right]=\left[x_{\alpha}, x_{\alpha}\right](m+n)+\left\langle x_{\alpha}, x_{\alpha}\right\rangle m \delta_{m+n, 0}=0 .
$$

By (3.8), this means that

$$
\left[Y\left(x_{\alpha}(-1) \mathbf{1}, x_{1}\right), Y\left(x_{\alpha}(-1) \mathbf{1}, x_{2}\right)\right]=0 .
$$

Thus for any $k \geq 0$, the product $Y\left(x_{\alpha}(-1) \mathbf{1}, x\right)^{k}$ is well-defined and equals the normalordered product ${ }_{\circ} Y\left(x_{\alpha}(-1) \mathbf{1}, x\right)^{k}$ 。 $($ see [LL] (3.8.4)). Then we can apply Proposition 3.10.2 in $[\mathrm{LL}$ to conclude that

$$
\begin{aligned}
Y\left(\frac{x_{\alpha}(-1)^{k}}{k !} \mathbf{1}, x\right) & =\frac{Y\left(x_{\alpha}(-1) \mathbf{1}, x\right)^{k}}{k !} \\
& =\frac{1}{k !}\left(\sum_{n_{1} \in \mathbb{Z}} x_{\alpha}\left(n_{1}\right) x^{-n_{1}-1}\right) \cdots\left(\sum_{n_{k} \in \mathbb{Z}} x_{\alpha}\left(n_{k}\right) x^{-n_{k}-1}\right) \\
& =\frac{1}{k !} \sum_{l \in \mathbb{Z}}\left(\sum_{n_{1}+\cdots+n_{k}=l} x_{\alpha}\left(n_{1}\right) \cdots x_{\alpha}\left(n_{k}\right)\right) x^{-l-k}
\end{aligned}
$$

Consider the coefficient of $x^{-l-k}$ in (3.27) for any $l \in \mathbb{Z}$, that is,

$$
\frac{1}{k !} \sum_{n_{1}+\ldots+n_{k}=l} x_{\alpha}\left(n_{1}\right) \cdots x_{\alpha}\left(n_{k}\right)
$$

Since all the $x_{\alpha}(n)$ operators commute with each other, for any $\sigma \in S_{n}, x_{\alpha}\left(n_{1}\right) \cdots x_{\alpha}\left(n_{k}\right)=$ $x_{\alpha}\left(n_{\sigma(1)}\right) \cdots x_{\alpha}\left(n_{\sigma(k)}\right)$. Thus we can collect some of the terms in (3.28) . 
Take any "partition" of $l$ into exactly $k$ parts, where parts are allowed to be negative or zero, as well as positive. Suppose the distinct parts are $n_{1}, \ldots, n_{m}$ where $n_{j}$ occurs $i_{j}$ times, that is, $n_{j} \in \mathbb{Z}, n_{1} i_{1}+\ldots+n_{m} i_{m}=l$, and $i_{1}+\ldots+i_{m}=k$. The terms in the sum in (3.28) corresponding to this partition are $x_{\alpha}\left(n_{1}\right)^{i_{1}} \cdots x_{\alpha}\left(n_{m}\right)^{i_{m}}$ and all permutations. The number of distinct permutations is

$$
\left(\begin{array}{c}
k \\
i_{1}
\end{array}\right)\left(\begin{array}{c}
k-i_{1} \\
i_{2}
\end{array}\right) \cdots\left(\begin{array}{c}
k-i_{1}-\ldots-i_{m-1} \\
i_{m}
\end{array}\right)
$$

(note that $\left.\left(\begin{array}{c}k-i_{1}-\ldots-i_{m-1} \\ i_{m}\end{array}\right)=\left(\begin{array}{l}i_{m} \\ i_{m}\end{array}\right)=1\right)$. This equals

$$
\frac{k !}{\left(k-i_{1}\right) ! i_{1} !} \frac{\left(k-i_{1}\right) !}{\left(k-i_{1}-i_{2}\right) ! i_{2} !} \cdots 1=\frac{k !}{i_{1} ! i_{2} ! \cdots i_{m} !} .
$$

Hence the sum of all terms in (3.28) corresponding to this partition is

$$
\frac{1}{k !} \frac{k !}{i_{1} ! i_{2} ! \cdots i_{m} !} x_{\alpha}\left(n_{1}\right)^{i_{1}} \cdots x_{\alpha}\left(n_{m}\right)^{i_{m}}=\frac{x_{\alpha}\left(n_{1}\right)^{i_{1}}}{i_{1} !} \cdots \frac{x_{\alpha}\left(n_{m}\right)^{i_{m}}}{i_{m} !} .
$$

Thus the coefficient of $x^{-l-k}$ in $Y\left(x_{\alpha}(-1) \mathbf{1}, x\right)^{k} / k$ ! is

$$
\sum_{\substack{\text { partitions of } l \\ \text { with } k \text { parts }}} \frac{x_{\alpha}\left(n_{1}\right)^{i_{1}}}{i_{1} !} \cdots \frac{x_{\alpha}\left(n_{m}\right)^{i_{m}}}{i_{m} !} .
$$

Considering the case $U=\mathbb{C} 1$, it is clear from (3.23), (3.24) and (3.32) that the vertex subring generated by the $\frac{x_{\alpha}(-1)^{k}}{k !} \mathbf{1}$ is contained in $V_{\widehat{\mathfrak{g}}}(\ell, 0)_{\mathbb{Z}}$. On the other hand, we can use induction on $k$ to show that for any $m \in \mathbb{Z}$ and $\alpha$ a root of $\mathfrak{g}, x_{\alpha}(m)^{k} / k$ ! preserves the vertex subring generated by the $\frac{x_{\alpha}(-1)^{k}}{k !} \mathbf{1}$. This is trivially true for $k=0$. If $k>0$, take $l=m k$ in (3.32) to conclude that

$$
\frac{x_{\alpha}(m)^{k}}{k !}=\left(\frac{x_{\alpha}(-1)^{k}}{k !} \mathbf{1}\right)_{m k+k-1}-\sum_{\text {partitions } \neq(m, \ldots, m)} \frac{x_{\alpha}\left(n_{1}\right)^{i_{1}}}{i_{1} !} \cdots \frac{x_{\alpha}\left(n_{m}\right)^{i_{m}}}{i_{m} !} .
$$

Since each $i_{j}<k$ on the right side, by induction every term on the right side preserves the vertex subring, so $x_{\alpha}(m)^{k} / k$ ! does as well. Since 1 is in any vertex subring, (3.23) now implies that $V_{\widehat{\mathfrak{g}}}(\ell, 0)_{\mathbb{Z}}$ is contained in the vertex subring generated by the $\frac{x_{\alpha}(-1)^{k}}{k !} \mathbf{1}$. In the same way,

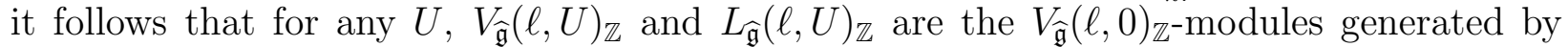
$U_{\mathbb{Z}}$

Corollary 3.6. The integral form $L_{\widehat{\mathfrak{g}}}(\ell, 0)_{\mathbb{Z}}$ of $L_{\widehat{\mathfrak{g}}}(\ell, 0)$ is the integral form of $L_{\widehat{\mathfrak{g}}}(\ell, 0)$ as a vertex algebra generated by the vectors $\frac{x_{\alpha}(-1)^{k}}{k !} \mathbf{1}$ where $\alpha$ is a root and $k \geq 0$.

Proof. We know from the proof of Theorem 3.5 that $L_{\widehat{\mathfrak{g}}}(\ell, 0)_{\mathbb{Z}}$ is spanned by coefficients of products of the form in (3.24), but by Proposition 2.1, this is precisely the vertex subring of $L_{\widehat{\mathfrak{g}}}(\ell, 0)$ generated by the $\frac{x_{\alpha}(-1)^{k}}{k !} \mathbf{1}$. Note that although the $Y$ in $(\underline{3.24})$ is the vertex operator for $V_{\widehat{\mathfrak{g}}}(\ell, 0)$ acting on $L_{\widehat{\mathfrak{g}}}(\ell, 0)$, the vertex operator for $L_{\widehat{\mathfrak{g}}}(\ell, 0)$ acting on itself is defined the same way. 
Corollary 3.7. If $\mathfrak{g}$ is of type $A, D$, or $E$ and $\ell$ is a positive integer, the integral form $L_{\widehat{\mathfrak{g}}}(\ell, 0)_{\mathbb{Z}}$ of $L_{\widehat{\mathfrak{g}}}(\ell, 0)$ is generated by the vectors $\frac{x_{\alpha}(-1)^{k}}{k !} \mathbf{1}$ where $\alpha$ is a root of $\mathfrak{g}$ and $0 \leq k \leq \ell$.

Proof. This follows from the well-known fact that for any long root $\alpha, x_{\alpha}(-1)^{\ell+1} \cdot v_{0}=$ 0 , where $v_{0}$ is a highest weight vector of a standard level $\ell \widehat{\mathfrak{g}}$-module (see for example Proposition 6.6.4 in [LL]).

Remark 3.8. The integral form $L_{\widehat{\mathfrak{g}}}(\ell, 0)_{\mathbb{Z}}$ as in Corollary 3.7 is generally larger than the one constructed using the integral form of $U(\widehat{\mathfrak{g}})$ discussed in Remark 3.1, although they coincide when $\mathfrak{g}$ is a finite-dimensional simply-laced simple Lie algebra and $\ell=1$.

\section{Integral forms for lattice vertex algebras}

We recall the construction of conformal vertex algebras from even lattices. Suppose $L$ is a non-degenerate even lattice with form $\langle\cdot, \cdot\rangle$. Consider also the space $\mathfrak{h}=\mathbb{C} \otimes_{\mathbb{Z}} L$, an abelian Lie algebra with a (trivially) invariant form. Thus we can form the Heisenberg vertex operator algebra $V_{\widehat{\mathfrak{h}}}(1,0)$, which is linearly isomorphic to $S\left(\widehat{\mathfrak{h}}_{-}\right)$, the symmetric algebra on $\widehat{\mathfrak{h}}_{-}$. We can also construct the twisted group algebra $\mathbb{C}\{L\}$ as follows: given a positive integer $s$, take a central extension of $L$ by the cyclic group of $s$ elements:

$$
1 \rightarrow\left\langle\kappa \mid \kappa^{s}=1\right\rangle \rightarrow \widehat{L} \rightarrow L \rightarrow 1 .
$$

If $\omega_{s}$ is a primitive $s$ th root of unity, let $\mathbb{C}_{\omega_{s}}$ denote the one-dimensional $\langle\kappa\rangle$-module on which $\kappa$ acts as $\omega_{s}$. Then the twisted group algebra is the induced $\widehat{L}$-module

$$
\mathbb{C}\{L\}=\mathbb{C}[\widehat{L}] \otimes_{\mathbb{C}[\langle\kappa\rangle]} \mathbb{C}_{\omega_{s}}
$$

It is linearly isomorphic to the group algebra $\mathbb{C}[L]$. We need to choose the integer $s$ and the central extension so that the commutator map $c_{0}$, defined by the condition $a b=b a \kappa^{c_{0}(\bar{a}, \bar{b})}$ for $a, b \in \widehat{L}$, satisfies the condition

$$
\omega_{s}^{c_{0}(\alpha, \beta)}=(-1)^{\langle\alpha, \beta\rangle}
$$

for $\alpha, \beta \in L$. For instance, since $L$ is even, we can take $s=2$ and $c_{0}(\alpha, \beta)=\langle\alpha, \beta\rangle(\bmod 2)$. (The resulting vertex operator algebra does not depend up to isomorphism on the choices of $s$ and the central extension; see [LL], Proposition 6.5.5.) The $\widehat{L}$-module $\mathbb{C}\{L\}$ is also a module for $\mathfrak{h}=\mathfrak{h} \otimes t^{0}:$

$$
h(0)(a \otimes 1)=\langle h, \bar{a}\rangle(a \otimes 1)
$$

for $h \in \mathfrak{h}$ and $a \in \widehat{L}$. Also, for $\alpha \in L$ and $x$ a formal variable, define a map $x^{\alpha}$ on $\mathbb{C}\{L\}$ by

$$
x^{\alpha}(b \otimes 1)=(b \otimes 1) x^{\langle\alpha, \bar{b}\rangle}
$$

for $b \in \widehat{L}$.

We can now extend the vertex operator algebra structure on $S\left(\widehat{\mathfrak{h}}_{-}\right)$to the larger space

$$
V_{L}=S\left(\widehat{\mathfrak{h}}_{-}\right) \otimes \mathbb{C}\{L\}
$$


For $a \in \widehat{L}$, use $\iota(a)$ to denote the element $1 \otimes(a \otimes 1) \in V_{L}$. As a vertex algebra, $V_{L}$ is generated by the $\iota(a)$, which have vertex operators

$$
Y(\iota(a), x)=E^{-}(-\bar{a}, x) E^{+}(-\bar{a}, x) a x^{\bar{a}}
$$

where

$$
E^{ \pm}(\alpha, x)=\exp \left(\sum_{n \in \pm \mathbb{Z}_{+}} \frac{\alpha(n)}{n} x^{-n}\right)
$$

for any $\alpha \in L$, and $a$ denotes the action of $a \in \widehat{L}$ on $\mathbb{C}\{L\}$. The conformal element of $V_{L}$ is the same as the conformal element in the Heisenberg algebra:

$$
\omega=\frac{1}{2} \sum_{i=1}^{\operatorname{dim} \mathfrak{h}} \alpha_{i}(-1) \alpha_{i}^{\prime}(-1) \mathbf{1},
$$

where $\left\{\alpha_{i}\right\}$ is a basis for $\mathfrak{h}$ and $\left\{\alpha_{i}^{\prime}\right\}$ is the corresponding dual basis with respect to $\langle\cdot, \cdot\rangle$. If $L$ is positive definite, $V_{L}$ is a vertex operator algebra in the sense that the finiteness restrictions on the weight spaces hold. If $L$ is not positive definite, $V_{L}$ is still a strongly $L$-graded conformal vertex algebra, where for $\alpha \in L$,

$$
V^{\alpha}=S\left(\widehat{\mathfrak{h}}_{-}\right) \otimes \iota(a),
$$

with $a \in \widehat{L}$ such that $\bar{a}=\alpha$.

To obtain modules for $V_{L}$, consider $L^{\circ}$, the dual lattice of $L$, and construct the space

$$
V_{L^{\circ}}=S\left(\widehat{\mathfrak{h}}_{-}\right) \otimes \mathbb{C}\left\{L^{\circ}\right\}
$$

in the same way we constructed $V_{L}$. In particular, we need a central extension of $L^{\circ}$ by $\left\langle\kappa \mid \kappa^{s}=1\right\rangle, s$ an even integer, with commutator map $c_{0}$, having the property that

$$
\omega_{s}^{c_{0}(\alpha, \beta)}=(-1)^{\langle\alpha, \beta\rangle}
$$

for $\alpha, \beta \in L$, where $\omega_{s}$ is the sth root of unity used to construct $\mathbb{C}\left\{L^{\circ}\right\}$. Such a central extension always exists (see Remark 6.4.12 in [LL]).

Then $V_{L^{\circ}}$ is a $V_{L^{-}}$-module with the same action of $\iota(a)$ as in (4.7). For any $S \subseteq L^{\circ}$, denote by $\mathbb{C}\{S\}$ the subspace of $\mathbb{C}\left\{L^{\circ}\right\}$ spanned by elements of the form $a \otimes 1$ where $\bar{a} \in S$. Then the spaces

$$
V_{\beta+L}=S\left(\widehat{\mathfrak{h}}_{-}\right) \otimes \mathbb{C}\{\beta+L\},
$$

where $\beta$ runs over coset representatives of $L^{\circ} / L$, exhaust the irreducible $V_{L}$-modules up to equivalence.

The vertex algebra $V_{L}$ (repectively, the module $V_{L^{\circ}}$ ) is spanned by vectors of the form form

$$
\alpha_{1}\left(-n_{1}\right) \cdots \alpha_{k}\left(-n_{k}\right) \iota(b)
$$

where $\alpha_{i} \in L, n_{i} \in \mathbb{Z}_{+}$, and $\bar{b} \in L$ (respectively, $\bar{b} \in L^{\circ}$ ). Such a vector has conformal weight

$$
n_{1}+\ldots+n_{k}+\frac{\langle\bar{b}, \bar{b}\rangle}{2} \in \mathbb{Q} .
$$


The modules $V_{L^{\circ}}$ and $V_{\beta+L}$ where $\beta \in L^{\circ}$ are modules for $V_{L}$ as strongly $L$-graded conformal vertex algebra since they are graded by $L^{\circ}$ :

$$
V_{L^{\circ}}=\coprod_{\gamma \in L^{\circ}} V^{\gamma}
$$

where $V^{\gamma}=S\left(\widehat{\mathfrak{h}}_{-}\right) \otimes \iota(c), c \in L^{\circ}$ such that $\bar{c}=\gamma$, and $V_{\beta+L}$ is analogously graded.

In order to obtain integral structure in $V_{L}$ and its modules, we first need to show that we can choose the central extension so that we have integral structure in the twisted group algebra $\mathbb{C}\{L\}$. Given a central extension $\widehat{L^{\circ}}$ of $L^{\circ}$, we can choose a section $L^{\circ} \rightarrow \widehat{L^{\circ}}$, denoted $\beta \mapsto e_{\beta}$ for $\beta \in L^{\circ}$. We define a 2-cocycle $\varepsilon_{0}: L^{\circ} \times L^{\circ} \rightarrow \mathbb{Z} / s \mathbb{Z}$ by

$$
e_{\alpha} e_{\beta}=e_{\alpha+\beta} \kappa^{\varepsilon_{0}(\alpha, \beta)} .
$$

Conversely, given a 2-cocycle (such as a bilinear map) $\varepsilon_{0}: L^{\circ} \times L^{\circ} \rightarrow \mathbb{Z} / s \mathbb{Z}$ we can define a central extension of $L^{\circ}$ by $\left\langle\kappa \mid \kappa^{s}=1\right\rangle$ (see Proposition 5.1.2 in [FLM]). Given an $\varepsilon_{0}$, define $\varepsilon(\alpha, \beta)=\omega_{s}^{\varepsilon_{0}(\alpha, \beta)}$. The following lemma is an adjustment of Remark 6.4.12 in [LL] (see also Remark 12.17 in [DL]):

Lemma 4.1. There exists a central extension $\widehat{L^{\circ}}$ satisfying $\sqrt{4.12)}$ and a section $L^{\circ} \rightarrow \widehat{L^{\circ}}$ such that $\varepsilon(\alpha, \beta)= \pm 1$ for any $\alpha, \beta \in L$.

Proof. It is possible to choose a base $\left\{\alpha_{1}, \ldots, \alpha_{l}\right\}$ of $L^{\circ}$ so that $\left\{n_{1} \alpha_{1}, \ldots, n_{l} \alpha_{l}\right\}$, where the $n_{i}$ are positive integers, forms a base for $L$. Since $\langle\cdot, \cdot\rangle$ is $\mathbb{Q}$-valued on $L^{\circ}$, there is a positive even integer $s$ such that $\frac{s}{2}\langle\alpha, \beta\rangle \in \mathbb{Z}$ for any $\alpha, \beta \in L^{\circ}$. Then we have the bilinear 2-cocycle $\varepsilon_{0}: L^{\circ} \times L^{\circ} \rightarrow \mathbb{Z} / s \mathbb{Z}$ determined by its values on the base:

$$
\varepsilon_{0}\left(\alpha_{i}, \alpha_{j}\right)=\left\{\begin{array}{cl}
\frac{s}{2}\left\langle\alpha_{i}, \alpha_{j}\right\rangle+s \mathbb{Z} & \text { if } i<j \\
0 & \text { if } i \geq j
\end{array} .\right.
$$

Using bilinearity, we have for $i<j$

$$
\varepsilon_{0}\left(n_{i} \alpha_{i}, n_{j} \alpha_{j}\right)=n_{i} n_{j} \varepsilon_{0}\left(\alpha_{i}, \alpha_{j}\right)=n_{i} n_{j} \frac{s}{2}\left\langle\alpha_{i}, \alpha_{j}\right\rangle+s \mathbb{Z}=\frac{s}{2}\left\langle n_{i} \alpha_{i}, n_{j} \alpha_{j}\right\rangle+s \mathbb{Z} .
$$

Since $\langle\cdot, \cdot\rangle$ is $\mathbb{Z}$-valued and bilinear on $L$, it follows that $\varepsilon(\alpha, \beta)= \pm 1$ for $\alpha, \beta \in L$.

The 2-cocycle $\varepsilon_{0}$ corresponds to a section of a central extension $\widehat{L^{\circ}}$ of $L^{\circ}$. The corresponding commutator map $c_{0}$ is given by

$$
c_{0}(\alpha, \beta)=\varepsilon_{0}(\alpha, \beta)-\varepsilon_{0}(\beta, \alpha)
$$

for $\alpha, \beta \in L^{\circ}$ since

$$
e_{\alpha} e_{\beta}=e_{\alpha+\beta} \kappa^{\varepsilon_{0}(\alpha, \beta)}=e_{\beta} e_{\alpha} \kappa^{-\varepsilon_{0}(\beta, \alpha)} \kappa^{\varepsilon_{0}(\alpha, \beta)} .
$$

Thus the commutator map is an alternating $\mathbb{Z}$-bilinear form $L^{\circ} \times L^{\circ} \rightarrow \mathbb{Z} / s \mathbb{Z}$. Since

$$
c_{0}\left(n_{i} \alpha_{i}, n_{j} \alpha_{j}\right)=\frac{s}{2}\left\langle n_{i} \alpha_{i}, n_{j} \alpha_{j}\right\rangle+s \mathbb{Z}
$$


for $i<j$ and since

$$
(\alpha, \beta) \mapsto \frac{s}{2}\langle\alpha, \beta\rangle+s \mathbb{Z}
$$

is also an alternating bilinear form on $L$ (since $L$ is even), it follows that

$$
c_{0}(\alpha, \beta)=\frac{s}{2}\langle\alpha, \beta\rangle+s \mathbb{Z}
$$

for all $\alpha, \beta \in L$. Thus

$$
\omega_{s}^{c_{0}(\alpha, \beta)}=\omega_{s}^{s\langle\alpha, \beta\rangle / 2}=(-1)^{\langle\alpha, \beta\rangle}
$$

for $\alpha, \beta \in L$, as required.

Throughout the rest of this section we will use the central extension and section of Lemma 4.1. Moreover, we will assume that $e_{0}=1$, so that $\iota\left(e_{0}\right)=1$. Thus $V_{L}$ is generated as vertex algebra by the $\iota\left(e_{\alpha}\right)$ for $\alpha \in L$. This motivates the following definition:

Definition 4.2. Define $V_{L, \mathbb{Z}}$ to be the vertex subring of $V_{L}$ generated by the $\iota\left(e_{\alpha}\right)$ for $\alpha \in L$. Moreover, for any $\beta \in L^{\circ}$, set $V_{\beta+L, \mathbb{Z}}$ equal to the $V_{L, \mathbb{Z}}$ submodule of $V_{\beta+L}$ generated by $\iota\left(e_{\beta}\right)$. In particular, $V_{L, \mathbb{Z}}$ itself is the $V_{L, \mathbb{Z}}$ submodule of $V_{L}$ generated by $\mathbf{1}$.

Then we have the following theorem (the algebra part is originally due to Borcherds $[\mathrm{B}]$ and has also been proved using a different method in $[\mathrm{P}]$ and $[\mathrm{DG}])$ :

Theorem 4.3. The vertex subring $V_{L, \mathbb{Z}}$ is an integral form of $V_{L}$, and for any $\beta \in L^{\circ}, V_{\beta+L, \mathbb{Z}}$ is an integral form of $V_{\beta+L}$.

Proof. Since $V_{L, \mathbb{Z}}$ is closed under vertex algebra products by definition, we just need to show that for any $\beta \in L^{\circ}, V_{\beta+L, \mathbb{Z}}$ is an integral form of the vector space $V_{\beta+L}$ and that $V_{\beta+L, \mathbb{Z}}$ is compatible with the $L^{\circ} \times \mathbb{Q}$-gradation of $V_{\beta+L}$, as in (2.7). By Proposition 2.1, $V_{\beta+L, \mathbb{Z}}$ is the $\mathbb{Z}$-span of coefficients of products of the form

$$
Y\left(\iota\left(e_{\alpha_{1}}\right), x_{1}\right) \ldots Y\left(\iota\left(e_{\alpha_{k}}\right), x_{k}\right) \iota\left(e_{\beta}\right)
$$

where $\alpha_{i} \in L$. Since $\iota\left(e_{\beta}\right)$ and $\iota\left(e_{\alpha}\right)$ for $\alpha \in L$ are homogeneous in the $L^{\circ} \times \mathbb{Q}$-gradation of $V_{L^{\circ}}$, it follows from (2.3) that coefficients of products as in (4.26) are doubly homogeneous. Hence $V_{\beta+L, \mathbb{Z}}$ is compatible with the $L^{\circ} \times \mathbb{Q}$-gradation:

$$
V_{\beta+L, \mathbb{Z}}=\coprod_{\gamma \in \beta+L, n \in \mathbb{Q}} V_{n}^{\gamma} \cap V_{\beta+L, \mathbb{Z}} .
$$

To show that $V_{\beta+L, \mathbb{Z}}$ is an integral form of the vector space $V_{\beta+L}$, it is enough to show that for any $\gamma \in \beta+L, V_{n}^{\gamma} \cap V_{\beta+L, \mathbb{Z}}$ is a lattice in $V_{n}^{\gamma}$ whose rank is the dimension of $V_{n}^{\gamma}$. Since the vectors $\iota\left(e_{\alpha}\right)$ for $\alpha \in L$ generate $V_{L}$ as a vertex algebra and since $\iota\left(e_{\beta}\right)$ generates $V_{\beta+L}$ as a $V_{L}$-module, $V_{\beta+L, \mathbb{Z}}$ spans $V_{\beta+L}$ over $\mathbb{C}$, and thus by (4.27), $V_{n}^{\gamma} \cap V_{\beta+L, \mathbb{Z}}$ spans $V_{n}^{\gamma}$ over $\mathbb{C}$ for any $\gamma \in \beta+L$. Thus if $V_{n}^{\gamma} \cap V_{\beta+L, \mathbb{Z}}$ is a lattice, its rank is at least the dimension of $V_{n}^{\gamma}$.

On the other hand, since $\varepsilon(\alpha, \beta)= \pm 1 \in \mathbb{Q}$ for any $\alpha, \beta \in L, V_{L}$ and $V_{\beta+L}$ have $\mathbb{Q}$ forms, namely, the $\mathbb{Q}$-subalgebra $V_{L, \mathbb{Q}} \subseteq V_{L}$ generated by the $\iota\left(e_{\alpha}\right)$ for $\alpha \in L$, and the 
$V_{L, \mathbb{Q}}$-submodule $V_{\beta+L, \mathbb{Q}}$ generated by $\iota\left(e_{\beta}\right)$, respectively. They are spanned over $\mathbb{Q}$ by the vectors

$$
\alpha_{1}\left(-n_{1}\right) \cdots \alpha_{k}\left(-n_{k}\right) \iota\left(e_{\gamma}\right)
$$

where $\alpha_{i} \in L, n_{i} \in \mathbb{Z}_{+}$, and $\gamma \in L$ or $\gamma \in \beta+L$, respectively. Now, any set of vectors in $V_{n}^{\gamma} \cap V_{\beta+L, \mathbb{Z}}$ which is linearly independent over $\mathbb{Z}$ is linearly independent over $\mathbb{Q}$ since a dependence relation over $\mathbb{Q}$ reduces to a dependence relation over $\mathbb{Z}$ by clearing denominators. Thus, since $V_{L, \mathbb{Z}} \subseteq V_{L, \mathbb{Q}}$, any set of vectors in $V_{n}^{\gamma} \cap V_{\beta+L, \mathbb{Z}}$ which is linearly independent over $\mathbb{Z}$ is linearly independent over $\mathbb{C}$. This means that if $V_{n}^{\gamma} \cap V_{\beta+L, \mathbb{Z}}$ is a lattice, its rank is no more than the dimension of $V_{n}^{\gamma}$.

Thus we are reduced to showing that for any $\gamma \in \beta+L, V_{n}^{\gamma} \cap V_{\beta+L, \mathbb{Z}}$ is a lattice in $V_{n}^{\gamma}$, that is, it is spanned over $\mathbb{Z}$ by a finite set. To show this, we use formula (8.4.22) in [FLM] to obtain

$$
\begin{aligned}
Y\left(\iota\left(e_{\alpha_{1}}\right), x_{1}\right) \cdots Y\left(\iota\left(e_{\alpha_{k}}\right), x_{k}\right) \iota\left(e_{\beta}\right)= & { }_{\circ} Y\left(\iota\left(e_{\alpha_{1}}\right), x_{1}\right) \cdots Y\left(\iota\left(e_{\alpha_{k}}\right), x_{k}\right){ }_{\circ} \iota\left(e_{\beta}\right) . \\
& \prod_{1 \leq i<j \leq k}\left(x_{i}-x_{j}\right)^{\left\langle\alpha_{i}, \alpha_{j}\right\rangle} .
\end{aligned}
$$

where $\alpha_{i} \in L$. (See also formula (8.6.6) in [FLM]; we use the normal ordering notation of [FLM] here.) Since $L$ is even and in particular integral, the binomial product expansions on the right side involve only integer coefficients. Hence coefficients of the non-normal ordered product are integral combinations of coefficients of the corresponding normal ordered product, and vice versa since the coefficients of

$$
\prod_{1 \leq i<j \leq k}\left(x_{i}-x_{j}\right)^{-\left\langle\alpha_{i}, \alpha_{j}\right\rangle}
$$

are also integers. This shows that we can define $V_{\beta+L, \mathbb{Z}}$ as the $\mathbb{Z}$-span of coefficients in products of the form

$$
{ }_{\circ}^{\circ} Y\left(\iota\left(e_{\alpha_{1}}\right), x_{1}\right) \cdots Y\left(\iota\left(e_{\alpha_{k}}\right), x_{k}\right){ }_{\circ} \iota\left(e_{\beta}\right)
$$

where $\alpha_{i} \in L$. By the definition of normal ordering, (4.31) equals

$$
x_{1}^{\left\langle\alpha_{1}, \beta\right\rangle} \cdots x_{k}^{\left\langle\alpha_{k}, \beta\right\rangle} E^{-}\left(-\alpha_{1}, x_{1}\right) \cdots E^{-}\left(-\alpha_{k}, x_{k}\right) \iota\left(e_{\alpha_{1}} \cdots e_{\alpha_{k}} e_{\beta}\right) .
$$

Thus, because $\varepsilon(\alpha, \beta)= \pm 1$ for any $\alpha, \beta \in L, V_{\beta+L, \mathbb{Z}}$ is the $\mathbb{Z}$-span of coefficients in products of the form

$$
E^{-}\left(-\alpha_{1}, x_{1}\right) \cdots E^{-}\left(-\alpha_{k}, x_{k}\right) \iota\left(e_{\gamma}\right)
$$

where $\alpha_{i} \in L$ and $\gamma \in \beta+L$. In fact, if $\left\{\alpha^{(1)}, \ldots, \alpha^{(l)}\right\}$ is a base for $L$ (or any finite spanning set), we may take the $\alpha_{i}$ in (4.33) to come from $\left\{ \pm \alpha^{(1)}, \ldots, \pm \alpha^{(l)}\right\}$, since if $\alpha=\sum_{i=1}^{l} n_{i} \alpha^{(i)} \in$ $L$, then by properties of exponentials,

$$
E^{-}(-\alpha, x)=\prod_{i=1}^{l} E^{-}\left(-\alpha^{(i)}, x\right)^{n_{i}},
$$

where if $n_{i}$ is negative, $E^{-}\left(-\alpha^{(i)}, x\right)^{n_{i}}=E^{-}\left(\alpha^{(i)}, x\right)^{-n_{i}}$. 
Recall that for $\alpha \in L$,

$$
E^{-}(-\alpha, x)=\exp \left(\sum_{n>0} \frac{\alpha(-n)}{n} x^{n}\right),
$$

so the coefficient of $x^{m}$ in this operator increases weight by $m$. Hence the coefficient of any monomial of total degree $m$ in (4.33) is in $V_{m+\langle\gamma, \gamma\rangle / 2}^{\gamma}$. The coefficients of such monomials, with $\gamma$ fixed, for which $m+\langle\gamma, \gamma\rangle / 2=n \operatorname{span} V_{\beta+L, \mathbb{Z}} \cap V_{n}^{\gamma}$. Since $\left\{ \pm \alpha^{(1)}, \ldots, \pm \alpha^{(l)}\right\}$ is a finite set, there are only a finite number of ways of obtaining coefficients of products of the form (4.33) that lie in $V_{n}^{\gamma}$. This shows that $V_{\mathbb{Z}} \cap V_{n}^{\gamma}$ is finitely generated for any $n \in \mathbb{Q}, \gamma \in \beta+L$, completing the proof.

Remark 4.4. Borcherds' definition of $V_{L, \mathbb{Z}}$ in $[\mathrm{B}]$ does not use the vertex algebra structure of $V_{L}$; note that $V_{L}$ is also an associative algebra with product determined by

$$
\begin{array}{r}
\left(\alpha_{1}\left(-m_{1}\right) \cdots \alpha_{j}\left(-m_{j}\right) \iota(a)\right)\left(\beta_{1}\left(-n_{1}\right) \cdots \beta_{k}\left(-n_{k}\right) \iota(b)\right)= \\
\alpha_{1}\left(-m_{1}\right) \cdots \alpha_{j}\left(-m_{j}\right) \beta_{1}\left(-n_{1}\right) \cdots \beta_{k}\left(-n_{k}\right) \iota(a b),
\end{array}
$$

where $\alpha_{i}, \beta_{i}, \bar{a}, \bar{b} \in L, m_{i}, n_{i}>0$. Thus $V_{L}$ is generated as an associative algebra by the elements $\iota(a)$ for $\bar{a} \in L$ and $\alpha(-n) \mathbf{1}$ where $\alpha \in L$ and $n>0$. There is a derivation $D$ of this associative algebra structure defined on generators by $D \iota(a)=\bar{a}(-1) \iota(a)$ and $D \alpha(-n) \mathbf{1}=n \alpha(-n-1) \mathbf{1}$. This is precisely the action of $L(-1)$ on these elements, and in fact $D=L(-1)$. In $[\mathrm{B}], V_{L, \mathbb{Z}}$ is defined to be the smallest associative subring of $V_{L}$ containing each $\iota\left(e_{\alpha}\right)$ and invariant under $D^{i} / i$ ! for $i \geq 0$. It is claimed that $V_{L, \mathbb{Z}}$ is then generated as associative ring by the $\iota\left(e_{\alpha}\right)$ and the coefficients of $E^{-}(-\alpha, x) \mathbf{1}$, that is, $V_{L, \mathbb{Z}}$ is the $\mathbb{Z}$-span of coefficients of products of the form (4.33) (where $\gamma$ is now in $L$ ). From the proof of Theorem 4.3, we know that such vectors span $V_{L, \mathbb{Z}}$ as we have defined it here. Borcherds' claim has been proven in $[\mathrm{P}]$, but we simplify the proof here:

Proposition 4.5. The definition of $V_{L, \mathbb{Z}}$ in [B] agrees with Definition [4.2.

Proof. Let $V_{L, \mathbb{Z}}^{*}$ denote the structure defined in [B], and $V_{L, \mathbb{Z}}$ the structure of Definition 4.2. First, we show $V_{L, \mathbb{Z}} \subseteq V_{L, \mathbb{Z}}^{*}$. Since $V_{L, \mathbb{Z}}$ is the $\mathbb{Z}$-span of coefficients of products of the form (4.33) and $V_{L, \mathbb{Z}}^{*}$ is an associative subring, it is enough to show that each $\iota\left(e_{\alpha}\right)$ for $\alpha \in L$ and the coefficients of each $E^{-}(-\alpha, x) \mathbf{1}$ for $\alpha \in L$ are in $V_{L, \mathbb{Z}}^{*}$. Now, each $\iota\left(e_{\alpha}\right) \in V_{L, \mathbb{Z}}^{*}$ by definition; also $V_{L, \mathbb{Z}}^{*}$ is closed under $L(-1)^{i} / i$ ! for each $i \geq 0$. Thus for any $\alpha \in L, V_{L, \mathbb{Z}}^{*}$ must contain the coefficients of

$$
e^{L(-1) x} \iota\left(e_{\alpha}\right)=Y\left(\iota\left(e_{\alpha}\right), x\right) \mathbf{1}=E^{-}(-\alpha, x) \iota\left(e_{\alpha}\right) .
$$

Recall that in any conformal vertex algebra, $e^{L(-1) x} v=Y(v, x) \mathbf{1}$ for any $v$ (formulas (3.1.29) and (3.1.67) in $[\mathrm{LL}]$ ). Since $V_{L, \mathbb{Z}}^{*}$ is an associative subring, it contains the coefficients of

$$
\left(E^{-}(-\alpha, x) \iota\left(e_{\alpha}\right)\right)\left(\iota\left(e_{-\alpha}\right)\right)= \pm E^{-}(-\alpha, x) \mathbf{1}
$$

since $\varepsilon(\alpha,-\alpha)= \pm 1$. 
On the other hand, $V_{L, \mathbb{Z}}$ is an associative subring of $V_{L}$ (the associative product of any two coefficients of products of the form (4.33) is again such a coefficient). Also, $V_{L, \mathbb{Z}}$ is invariant under each $L(-1)^{i} / i$ ! since it is closed under vertex operators and $e^{L(-1) x} v=Y(v, x) \mathbf{1}$ for $v \in V_{L, \mathbb{Z}}$. Thus $V_{L, \mathbb{Z}}^{*} \subseteq V_{L, \mathbb{Z}}$, and $V_{L, \mathbb{Z}}=V_{L, \mathbb{Z}}^{*}$.

Remark 4.6. If $L$ is the root lattice of a finite-dimensional simple Lie algebra $\mathfrak{g}$ of type $A$, $D$, or $E$, the lattice vertex operator algebra $V_{L}$ is isomorphic to the level 1 affine Lie algebra vertex operator algebra $L_{\widehat{\mathfrak{g}}}(1,0)$. The isomorphism is determined by

$$
\iota\left(e_{\alpha}\right) \mapsto \pm x_{\alpha}(-1) \mathbf{1}
$$

for $\alpha$ a root of $\mathfrak{g}$ and $x_{\alpha}$ the corresponding root vector in a Chevalley basis for $\mathfrak{g}$. (For the proof of this result see [FLM] and [DL.) From the definitions, it is clear the integral forms $V_{L, \mathbb{Z}}$ and $L_{\widehat{\mathfrak{g}}}(1,0)_{\mathbb{Z}}$ correspond under this isomorphism.

The following result on a $\mathbb{Z}$-base for $V_{\beta+L, \mathbb{Z}}$ has been proved for the algebra case $\beta=0$ in [DG], but since we will need it later, we include the proof for completeness; assume now that $\left\{\alpha^{(1)}, \ldots \alpha^{(l)}\right\}$ is a base for $L$ :

Proposition 4.7. The distinct coefficients of monomials in products as in (4.33) form a basis for $V_{\beta+L, \mathbb{Z}}$, where the $\alpha_{i}$ come from $\left\{\alpha^{(1)}, \ldots, \alpha^{(l)}\right\}$ and $\gamma$ is any element of $\beta+L$.

Proof. The proof of Theorem 4.3 shows that the coefficients of monomials in (4.33) span $V_{\beta+L, \mathbb{Z}}$ when the $\alpha_{i}$ come from $\left\{ \pm \alpha^{(1)}, \ldots, \pm \alpha^{(l)}\right\}$. However, recall that $E^{-}\left(\alpha^{(i)}, x\right)=$ $E^{-}\left(-\alpha^{(i)}, x\right)^{-1}$. If we expand

$$
E^{-}\left(-\alpha^{(i)}, x\right)=1+\sum_{j \geq 1} y_{i j} x^{j}
$$

where $y_{i j}$ is a polynomial in the $\alpha^{(i)}(-k)$, then

$$
E^{-}\left(\alpha^{(i)}, x\right)=\frac{1}{1+\sum_{j \geq 1} y_{i j} x^{j}}=\sum_{n \geq 0}\left(-\sum_{j \geq 1} y_{i j} x^{j}\right)^{n} .
$$

Thus the coefficients of $E^{-}\left(\alpha^{(i)}, x\right)$ are polynomials in the coefficients of $E^{-}\left(-\alpha^{(i)}, x\right)$. This shows that the coefficients of monomials in (4.33) span $V_{L^{\circ}, \mathbb{Z}}$ when the $\alpha_{i} \in\left\{\alpha^{(1)}, \ldots, \alpha^{(l)}\right\}$.

We also need to show that the indicated coefficients are linearly independent (over $\mathbb{Z}$ ). In fact, they are linearly independent over $\mathbb{C}$, and to show this, it is sufficient to show that the polynomials $y_{i j}$ are algebraically independent in $S\left(\widehat{\mathfrak{h}}_{-}\right)$. Since

$$
E^{-}\left(-\alpha^{(i)}, x\right)=\exp \left(\sum_{n<0} \frac{-\alpha^{(i)}(n)}{n} x^{-n}\right)=\exp \left(\sum_{n>0} \frac{\alpha^{(i)}(-n)}{n} x^{n}\right),
$$

$y_{i j}=\alpha^{(i)}(-j) / j+F_{i j}$, where $F_{i j}$ is a polynomial in the $\alpha^{(i)}(-k)$ with degree greater than 1 and with $k<j$.

Now suppose there is a relation

$$
F=\sum c_{i_{1} \ldots i_{k} ; j_{1} \ldots j_{k}} y_{i_{1} j_{1}} \cdots y_{i_{k} j_{k}}=0
$$


where all coefficients $c_{i_{1} \ldots i_{k} ; j_{1} \ldots j_{k}}$ are non-zero. If $k_{\text {min }}$ is the smallest degree of any monomial in the $y_{i j}$ in $F$, then the term of minimal degree in the $\alpha^{(i)}(-j)$ is

$$
\sum_{k=k_{m i n}} \frac{c_{i_{1} \ldots i_{k} ; j_{1} \ldots j_{k}}}{j_{1} \cdots j_{k}} \alpha^{\left(i_{1}\right)}\left(-j_{1}\right) \cdots \alpha^{\left(i_{k}\right)}\left(-j_{k}\right) .
$$

Since the $\alpha^{(i)}(-j)$ are algebraically independent, this sum must equal 0; but then each $c_{i_{1} \ldots i_{k} ; j_{1} \ldots j_{k}}=0$ for $k$ minimal as well. This contradiction shows that no nontrivial relation $F\left(\left\{y_{i j}\right\}\right)=0$ exists, so the $y_{i j}$ are algebraically independent.

Remark 4.8. We can express this basis for $V_{\beta+L, \mathbb{Z}}$ as the elements

$$
y_{i_{1} j_{1}} \cdots y_{i_{k} j_{k}} \iota\left(e_{\gamma}\right)
$$

where $k \geq 0,1 \leq i_{1} \leq \ldots \leq i_{k} \leq l, j_{m} \leq j_{m+1}$ if $i_{m}=i_{m+1}$, and $\gamma \in \beta+L$.

\section{The conformal vector in an integral form}

Suppose $A$ is an abelian group and $V$ is a strongly $A$-graded conformal vertex algebra with conformal vector $\omega$ and central charge $c \in \mathbb{C}$. Then we have the following result on when an integral form $V_{\mathbb{Z}}$ of $V$ can contain $\omega$ :

Proposition 5.1. If $V_{\mathbb{Z}}$ contains $k \omega$ where $k \in \mathbb{C}$, then $k^{2} c \in 2 \mathbb{Z}$.

Proof. If $k \omega \in V_{\mathbb{Z}}$, then $V_{\mathbb{Z}}$ must also contain

$$
(k L(2))\left(k(L(-2)) \mathbf{1}=k^{2} L(-2) L(2) \mathbf{1}+4 k^{2} L(0) \mathbf{1}+k^{2} \frac{c\left(2^{3}-2\right)}{12} \mathbf{1}=\frac{k^{2} c}{2} \mathbf{1} .\right.
$$

By Proposition 2.3, we must have $k^{2} c \in 2 \mathbb{Z}$.

In particular, the central charge of $V$ must be an even integer if $\omega$ is in any integral form of $V$. Now we prove a partial converse to Proposition 5.1. Recall that $v \in V$ is called a lowest weight vector for the Virasoro algebra if it is an $L(0)$-eigenvector and $L(n) v=0$ for $n>0$.

Theorem 5.2. Suppose $V_{\mathbb{Z}}$ is an integral form of $V$ generated by doubly homogeneous lowest weight vectors $\left\{v^{(j)}\right\}$ for the Virasoro algebra. If $k \in \mathbb{Z}$ is such that $k^{2} c \in 2 \mathbb{Z}$ and $k \omega \in V_{\mathbb{Q}}=$ $\mathbb{Q} \otimes_{\mathbb{Z}} V_{\mathbb{Z}}$, then $V_{\mathbb{Z}}$ can be extended to an integral form of $V$ containing $k \omega$.

Proof. We shall show that the vertex subring $V_{\mathbb{Z}}^{*}$ of $V$ generated by $V_{\mathbb{Z}}$ and $k \omega$ is an integral form of $V$. By Proposition 2.1, $V_{\mathbb{Z}}^{*}$ is spanned over $\mathbb{Z}$ by coefficients of products as in (2.8) where the $u_{i}$ are either $v^{(j)}$ or $k \omega$. Since the $v_{j}$ and $k \omega$ are homogeneous in the $A \times \mathbb{Z}_{\text {- }}$ gradation of $V$,

$$
V_{\mathbb{Z}}^{*}=\coprod_{\alpha \in A, n \in \mathbb{Z}} V_{\mathbb{Z}}^{*} \cap V_{n}^{\alpha}
$$

Since $k \omega \in V_{\mathbb{Q}}$ and $V_{\mathbb{Z}}^{*}$ contains $V_{\mathbb{Z}}$, which spans $V_{n}^{\alpha}$, it is enough to show that $V_{\mathbb{Z}}^{*} \cap V_{n}^{\alpha}$ is finitely generated as an abelian group, just as in the proof of Theorem 4.3. 
Lemma 5.3. For any $m, n \in \mathbb{Z}$ and lowest weight vector $v,\left[L(m), v_{n}\right]$ is an integral linear combination of operators $v_{k}$ for $k \in \mathbb{Z}$.

Proof. By the commutator formula,

$$
\begin{aligned}
{\left[Y\left(\omega, x_{1}\right), Y\left(v, x_{2}\right)\right] } & =\operatorname{Res}_{x_{0}} x_{2}^{-1} \delta\left(\frac{x_{1}-x_{0}}{x_{2}}\right) Y\left(Y\left(\omega, x_{0}\right) v, x_{2}\right) \\
& =\operatorname{Res}_{x_{0}} e^{-x_{0} \partial / \partial x_{1}}\left(x_{2}^{-1} \delta\left(\frac{x_{1}}{x_{2}}\right)\right) \sum_{n \in \mathbb{Z}} Y\left(L(n) v, x_{2}\right) x_{0}^{-n-2} \\
& =\sum_{n \geq-1}(-1)^{n+1}\left(\frac{\partial}{\partial x_{1}}\right)^{n+1}\left(x_{2}^{-1} \delta\left(\frac{x_{1}}{x_{2}}\right)\right) Y\left(L(n) v, x_{2}\right) .
\end{aligned}
$$

Since $v$ is a lowest weight vector, and using the $L(-1)$-derivative property,

$$
\begin{aligned}
{\left[Y\left(\omega, x_{1}\right), Y\left(v, x_{2}\right)\right]=} & x_{2}^{-1} \delta\left(\frac{x_{1}}{x_{2}}\right) \frac{d}{d x_{2}} Y\left(v, x_{2}\right) \\
& -(\operatorname{wt} v) \frac{\partial}{\partial x_{1}}\left(x_{2}^{-1} \delta\left(\frac{x_{1}}{x_{2}}\right)\right) Y\left(v, x_{2}\right)
\end{aligned}
$$

Since wt $v \in \mathbb{Z}$ and all the coefficients in the delta function expressions are integers, we see that $\left[L(m), v_{n}\right]$ is an integral combination of operators $v_{k}$.

Corollary 5.4. For $m \geq-1, L(m)$ leaves $V_{\mathbb{Z}}$ invariant.

Proof. By Lemma 5.3, an expression of the form $L(m) v_{n_{1}}^{\left(j_{1}\right)} \cdots v_{n_{k}}^{\left(j_{k}\right)} \mathbf{1}$ equals $v_{n_{1}}^{\left(j_{1}\right)} \cdots v_{n_{k}}^{\left(j_{k}\right)} L(m) \mathbf{1}$ plus an integral linear combination of terms of the form $v_{m_{1}}^{\left(j_{1}\right)} \cdots v_{m_{k}}^{\left(j_{k}\right)} \mathbf{1}$. But since $m \geq-1$, $L(m) \mathbf{1}=0$, so we see that $L(m) V_{\mathbb{Z}} \subseteq V_{\mathbb{Z}}$.

Continuing with the proof of Theorem 5.2, we can use Lemma 5.3 to rewrite any product of operators of the form $u_{m_{1}}^{(1)} \cdots u_{m_{k}}^{(k)}$, where the $u^{(i)}$ equal either $v^{(j)}$ or $k \omega$, as an integral combination of such products in which all operators of the form $k L(m)$ appear on the left. That is, $V_{\mathbb{Z}}^{*}$ is the integral span of products of the form

$$
\left(k L\left(m_{1}\right)\right) \cdots\left(k L\left(m_{j}\right)\right) v_{n_{1}}^{\left(j_{1}\right)} \cdots v_{n_{k}}^{\left(j_{k}\right)} \mathbf{1}
$$

where $m_{i}, n_{i} \in \mathbb{Z}$. We can now use the Virasoro algebra relations,

$$
[k L(m), k L(n)]=k(m-n)(k L(m+n))+\frac{k^{2} c\left(m^{3}-m\right)}{12} 1_{V},
$$

to rewrite $\left(k L\left(m_{1}\right)\right) \cdots\left(k L\left(m_{j}\right)\right)$ as an integral combination of such products for which $m_{1} \leq$ $\ldots \leq m_{j}$. (Note that since $k^{2} c \in 2 \mathbb{Z}$ and $\frac{m^{3}-m}{6}=\left(\begin{array}{c}m+1 \\ 3\end{array}\right), \frac{k^{2} c\left(m^{3}-m\right)}{12}$ is always an integer.)

Thus, using Corollary 5.4, we see that $V_{\mathbb{Z}}^{*}$ is the integral span of products of the form

$$
\left(k L\left(-m_{1}\right)\right) \cdots\left(k L\left(-m_{j}\right)\right) v
$$


where $m_{i} \geq 2$ and $v \in V_{\mathbb{Z}}$. To see that $V_{\mathbb{Z}}^{*} \cap V_{n}^{\alpha}$ is finitely generated, suppose $v_{1}, \ldots v_{l} \in V_{\mathbb{Z}}$ span $V_{\mathbb{Z}} \cap\left(\coprod_{i=N_{\alpha}}^{n} V_{i}^{\alpha}\right)$, where $V_{m}^{\alpha}=0$ for $m<N_{\alpha}$. Then $V_{\mathbb{Z}}^{*} \cap V_{n}^{\alpha}$ is spanned by some of the vectors of the form $\left(k L\left(-m_{1}\right)\right) \cdots\left(k L\left(-m_{j}\right)\right) v_{k}$ where $m_{i} \geq 2$ and $m_{1}+\ldots+m_{j} \leq$ $n-N_{\alpha}$. Since there are finitely many such vectors, $V_{\mathbb{Z}}^{*} \cap V_{n}^{\alpha}$ is finitely generated as an abelian group.

Proposition 5.5. Suppose $V_{\mathbb{Z}}$ is the integral form $V_{L, \mathbb{Z}}$ of a lattice vertex operator algebra $V_{L}$ or the integral form $V_{\widehat{\mathfrak{g}}}(\ell, 0)_{\mathbb{Z}}$ or $L_{\widehat{\mathfrak{g}}}(\ell, 0)_{\mathbb{Z}}$ of an affine Lie algebra vertex operator algebra associated to a finite dimensional simple Lie algebra $\mathfrak{g}$, with $\ell \in \mathbb{Z}$. Then $\omega \in V_{\mathbb{Q}}$ and $V_{\mathbb{Z}}$ is generated by lowest weight vectors for the Virasoro algebra, so $V_{\mathbb{Z}}$ may be extended to an integral form $V_{\mathbb{Z}}^{*}$ containing $k \omega$ for any $k \in \mathbb{Z}$ such that $k^{2} c \in 2 \mathbb{Z}$.

Proof. If $V=V_{L}$ and $\left\{\alpha_{1}, \ldots, \alpha_{l}\right\}$ is a base for $L$ with dual base $\left\{\alpha_{1}^{\prime}, \ldots, \alpha_{l}^{\prime}\right\}$ for $L^{\circ}$, then

$$
\omega=\frac{1}{2} \sum_{i=1}^{l} \alpha_{i}(-1) \alpha_{i}^{\prime}(-1) \mathbf{1}
$$

Since $\langle\cdot, \cdot\rangle$ is integral on $L, \alpha_{i}^{\prime} \in \mathbb{Q} \otimes_{\mathbb{Z}} L$ for any $1 \leq i \leq l$, so that $\omega \in V_{\mathbb{Q}}$. Moreover, $V_{\mathbb{Z}}$ is generated by the lowest weight vectors $\iota\left(e_{\alpha}\right)$ for $\alpha \in L$.

If $V$ is an affine Lie algebra vertex operator algebra $V_{\widehat{\mathfrak{g}}}(\ell, 0)_{\mathbb{Z}}$ or $L_{\widehat{\mathfrak{g}}}(\ell, 0)_{\mathbb{Z}}$ and $\left\{u_{i}\right\}$ is a Chevalley basis for $\mathfrak{g}_{\mathbb{Z}}$ with dual basis $\left\{u_{i}^{\prime}\right\}$ with respect to the form $\langle\cdot, \cdot\rangle$ on $\mathfrak{g}$, then

$$
\omega=\frac{1}{2(\ell+h)} \sum_{i=1}^{\operatorname{dim} \mathfrak{g}} u_{i}(-1) u_{i}^{\prime}(-1) \mathbf{1}
$$

where $h$ is the dual Coxeter number of $\mathfrak{g}$. Since $\ell, h \in \mathbb{Z}$ and $\langle\cdot, \cdot\rangle$ is integral on $\mathfrak{g}_{\mathbb{Z}}$ so that $u_{i}^{\prime} \in \mathbb{Q} \otimes_{\mathbb{Z}} \mathfrak{g}_{\mathbb{Z}}$, we have $\omega \in V_{\mathbb{Q}}$. Moreover, $V_{\mathbb{Z}}$ is generated by the vectors $\frac{x_{\alpha}(-1)^{k}}{k !} \mathbf{1}$ for $\alpha$ a root, $x_{\alpha}$ the corresponding root vector in the Chevalley basis of $\mathfrak{g}$, and $k \geq 0$. The commutation relations

$$
\left[L(m), x_{\alpha}(-1)\right]=x_{\alpha}(m-1)
$$

for any $m \in \mathbb{Z}$ (see for example [LL Section 6.2), and the fact that $x_{\alpha}(m-1)$ commutes with $x_{\alpha}(-1)$, imply that for $m>0$ and $k \geq 0$,

$$
L(m) \frac{x_{\alpha}(-1)^{k}}{k !} \mathbf{1}=\frac{x_{\alpha}(-1)^{k-1}}{(k-1) !} x_{\alpha}(m-1) \mathbf{1}=0 .
$$

Since $\frac{x_{\alpha}(-1)^{k}}{k !} \mathbf{1}$ is homogeneous of conformal weight $k$, this means $V_{\mathbb{Z}}$ is generated by lowest weight vectors for the Virasoro algebra.

Remark 5.6. Theorem 5.2 and Proposition 5.5 generalize the observation made in [B] that if $L$ is an even lattice, $V_{L, \mathbb{Z}}$ can be extended to an integral form of $V_{L}$ containing $\omega$ if the rank of $L$ is even, and containing $2 \omega$ if the rank of $L$ is odd.

Using the commutation relations (5.6), we can use an argument similar to but simpler than the proof of Theorem 5.2 to prove: 
Proposition 5.7. If $V$ is a vertex operator algebra generated by the conformal vector $\omega$, and $\omega$ is contained in a rational form of $V$, then $V$ has an integral form generated by $k \omega$ if $k \in \mathbb{Z}$ and $k^{2} c \in 2 \mathbb{Z}$. In particular, $\omega$ generates an integral form of $V$ if and only if $c \in 2 \mathbb{Z}$.

If $L$ is an even lattice, then $V_{L, \mathbb{Z}}$ may already contain $\omega$. Recall that if $\left\{\alpha_{1}, \ldots, \alpha_{l}\right\}$ is a base for $L$ and $\left\{\alpha_{1}^{\prime}, \ldots, \alpha_{l}^{\prime}\right\}$ is the corresponding dual basis with respect to $\langle\cdot, \cdot\rangle$, then

$$
\omega=\frac{1}{2} \sum_{i=1}^{l} \alpha_{i}(-1) \alpha_{i}^{\prime}(-1) \mathbf{1} \in V_{2}^{0},
$$

and the central charge of $V_{L}$ is $l$, the rank of $L$. The "if" direction of the following proposition was observed in [BR1]:

Proposition 5.8. If $L$ is an even lattice, the integral form $V_{L, \mathbb{Z}}$ of $V_{L}$ contains $\omega$ if and only if $L$ is self-dual.

Proof. Suppose $\left\{\alpha_{1}, \ldots, \alpha_{l}\right\}$ is a base for $L$. We know from Proposition 4.7 that an integral basis for $V_{L, \mathbb{Z}} \cap V_{2}^{0}$ consists of distinct coefficients of monomials of degree 2 in products of the form

$$
E^{-}\left(-\alpha_{i}, x_{1}\right) E^{-}\left(-\alpha_{j}, x_{2}\right) \mathbf{1}
$$

where $1 \leq i, j \leq l$. Since

$$
\begin{aligned}
E^{-}(-\alpha, x) & =\exp \left(\sum_{n>0} \frac{\alpha(-n)}{n} x^{n}\right) \\
& =1+\alpha(-1) x+\left(\frac{\alpha(-2)+\alpha(-1)^{2}}{2}\right) x^{2}+\ldots,
\end{aligned}
$$

the distinct coefficients of monomials of degree 2 in (15.13) are

$$
\alpha_{i}(-1) \alpha_{j}(-1) \mathbf{1}, \quad \frac{\alpha_{i}(-2)+\alpha_{i}(-1)^{2}}{2} \mathbf{1}
$$

where $1 \leq i \leq j \leq l$. We can take these quadratic polynomials as a base for $V_{L, \mathbb{Z}} \cap V_{2}^{0}$.

Now suppose $\left\{\alpha_{1}^{\prime}, \ldots, \alpha_{l}^{\prime}\right\}$ is a basis of $\mathfrak{h}$ dual to $\left\{\alpha_{1}, \ldots, \alpha_{l}\right\}$, and write $\alpha_{i}^{\prime}=\sum_{j=1}^{l} c_{j i} \alpha_{j}$ where $c_{j i} \in \mathbb{C}$. Then

$$
\begin{aligned}
\omega & =\frac{1}{2} \sum_{i=1}^{l} \alpha_{i}(-1) \alpha_{i}^{\prime}(-1) \mathbf{1}=\frac{1}{2} \sum_{i, j=1}^{l} c_{j i} \alpha_{i}(-1) \alpha_{j}(-1) \mathbf{1} \\
& =\sum_{i=1}^{l} \frac{c_{i i}}{2} \alpha_{i}(-1)^{2} \mathbf{1}+\sum_{i<j} \frac{c_{i j}+c_{j i}}{2} \alpha_{i}(-1) \alpha_{j}(-1) \mathbf{1}
\end{aligned}
$$

In view of the base (5.15) for $V_{L, \mathbb{Z}} \cap V_{2}^{0}$, we see that $\omega \in V_{L, \mathbb{Z}}$ if and only if $c_{i i}, c_{i j}+c_{j i} \in 2 \mathbb{Z}$ for all $i$ and $j \neq i$. 
Since $\left\{\alpha_{1}^{\prime}, \ldots, \alpha_{l}^{\prime}\right\}$ is a dual basis,

$$
\left\langle\alpha_{i}^{\prime}, \alpha_{j}^{\prime}\right\rangle=\left\langle\alpha_{i}^{\prime}, \sum_{k=1}^{l} c_{k j} \alpha_{k}\right\rangle=c_{i j}
$$

Since $\langle\cdot, \cdot\rangle$ is symmetric, we have $c_{i j}=c_{j i}$. Consequently, $\omega \in V_{\mathbb{Z}}$ if and only if $c_{i i} \in 2 \mathbb{Z}$ for all $i$ and $c_{i j} \in \mathbb{Z}$ for $i \neq j$. If $L$ is self-dual, each $\alpha_{i}^{\prime} \in L$, so each $c_{i j} \in \mathbb{Z}$; also, since $L$ is even, $c_{i i}=\left\langle\alpha_{i}^{\prime}, \alpha_{i}^{\prime}\right\rangle \in 2 \mathbb{Z}$ for each $i$. Conversely, if each $c_{i j} \in \mathbb{Z}$, then each $\alpha_{i}^{\prime} \in L$, so $L$ is self-dual. Thus we see that $\omega \in V_{L, \mathbb{Z}}$ if and only if $L$ is self-dual.

Example 5.9. If $L$ is the root lattice of $E_{8}$ or the Leech lattice, then $\omega \in V_{L, \mathbb{Z}}$.

\section{Integral forms in contragredient modules}

The following results generalize Lemma 6.1, Lemma 6.2, and Remark 6.3 in [DG] to the context of modules and contragredient modules for a vertex operator algebra $V$. Also, we apply these results to the affine Lie algebra vertex operator algebra integral forms $V_{\widehat{\mathfrak{g}}}(\ell, 0)_{\mathbb{Z}}$ and $L_{\widehat{\mathfrak{g}}}(\ell, 0)$, cases not considered in [DG. Suppose $V$ has an integral form $V_{\mathbb{Z}}$ and $W$ is a $V$-module with integral form $W_{\mathbb{Z}}$. Then there is an integral form $W_{\mathbb{Z}}^{\prime}$ in the contragredient module $W^{\prime}$ given by

$$
W_{\mathbb{Z}}^{\prime}=\left\{w^{\prime} \in W^{\prime} \mid\left\langle w^{\prime}, w\right\rangle \in \mathbb{Z} \text { for } w \in W_{\mathbb{Z}}\right\} .
$$

We would like $W_{\mathbb{Z}}^{\prime}$ to be a module for $V_{\mathbb{Z}}$.

Proposition 6.1. Suppose $V_{\mathbb{Z}}$ is invariant under $\frac{L(1)^{n}}{n !}$ for $n \geq 0$. Then $W_{\mathbb{Z}}^{\prime}$ is invariant under the action of $V_{\mathbb{Z}}$.

Proof. By the definition of the module action on $W^{\prime}$, if $v \in V_{\mathbb{Z}}, w \in W_{\mathbb{Z}}$ and $w^{\prime} \in W_{\mathbb{Z}}^{\prime}$,

$$
\left\langle Y(v, x) w^{\prime}, w\right\rangle=\left\langle w^{\prime}, Y\left(e^{x L(1)}\left(-x^{-2}\right)^{L(0)} v, x^{-1}\right) w\right\rangle
$$

Since $\frac{L(1)^{n}}{n !}$ leaves $V_{\mathbb{Z}}$ invariant, $e^{x L(1)}\left(-x^{-2}\right)^{L(0)} v \in V_{\mathbb{Z}}\left[x, x^{-1}\right]$. Since also $V_{\mathbb{Z}}$ leaves $W_{\mathbb{Z}}$ invariant, by definition $\left\langle Y(v, x) w^{\prime}, w\right\rangle \in \mathbb{Z}\left[\left[x, x^{-1}\right]\right]$ for any $w$ and so $Y(v, x) w^{\prime} \in W_{\mathbb{Z}}^{\prime}\left[\left[x, x^{-1}\right]\right]$ as desired.

Proposition 6.2. If $V_{\mathbb{Z}}$ is generated by vectors $v$ such that $L(1) v=0$, then $V_{\mathbb{Z}}$ is invariant under $\frac{L(1)^{n}}{n !}$ for $n \geq 0$.

Proof. We will use the $L(1)$-conjugation formula proved in [FHL]:

$$
e^{y L(1)} Y(v, x) e^{-y L(1)}=Y\left(e^{y(1-x y) L(1)}(1-x y)^{-2 L(0)} v, \frac{x}{1-x y}\right) .
$$

If $L(1) v=0$, this equation simplifies to

$$
e^{y L(1)} Y(v, x) e^{-y L(1)}=Y\left((1-x y)^{-2 L(0)} v, \frac{x}{1-x y}\right) .
$$


Thus if a vector $v \in V_{\mathbb{Z}}$ is a coefficient of a monomial in

$$
Y\left(v_{1}, x_{1}\right) \cdots Y\left(v_{k}, x_{k}\right) \mathbf{1}
$$

where $L(1) v_{i}=0$, then $\frac{L(1)^{n}}{n !} v$ is a coefficient of a monomial in

$$
\begin{aligned}
& e^{y L(1)} Y\left(v_{1}, x_{1}\right) \ldots Y\left(v_{k}, x_{k}\right) \mathbf{1}= \\
& Y\left(\left(1-x_{1} y\right)^{-2 L(0)} v_{1}, \frac{x_{1}}{1-x_{1} y}\right) \cdots Y\left(\left(1-x_{k} y\right)^{-2 L(0)} v_{k}, \frac{x_{k}}{1-x_{k} y}\right) \mathbf{1}
\end{aligned}
$$

Since the expansion of $(1-x y)^{m}$ for any integer $m$ has integer coefficients, all coefficients of monomials on the right side lie in $V_{\mathbb{Z}}$. Hence $\frac{L(1)^{n}}{n !} v \in V_{\mathbb{Z}}$. Thus if $V_{\mathbb{Z}}$ is spanned by coefficients of monomials of the form in (6.5), then $\frac{L(1)^{n}}{n !}$ leaves $V_{\mathbb{Z}}$ invariant for any $n \geq 0$.

Remark 6.3. If an integral form $V_{\mathbb{Z}}$ of $V$ is generated by lowest weight vectors for the

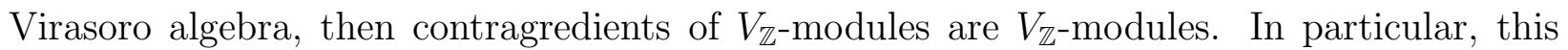
holds for lattice and affine Lie algebra vertex operator algebras by Proposition 5.5.

Suppose $V$ is equivalent as $V$-module to its contragredient $V^{\prime}$. This is the case if and only if there is a non-degenerate bilinear form $(\cdot, \cdot)$ on $V$ that is invariant in the sense that

$$
(Y(u, x) v, w)=\left(v, Y\left(e^{x L(1)}\left(-x^{-2}\right)^{L(0)} u, x^{-1}\right) w\right)
$$

for $u, v, w \in V$ (see [FHL]). Invariant forms on $V$ are in one-to-one correspondence with linear functionals on $V_{0} / L(1) V_{1}([\mathrm{Li}])$. In the case of lattice and affine Lie algebra vertex operator algebras, invariant forms are unique up to scale since $V_{0}=\mathbb{C} 1$ and $L(1) V_{1}=0$. (In the afffine Lie algebra case, $V$ has a non-degenerate invariant bilinear form only when $V=L_{\widehat{\mathfrak{g}}}(\ell, 0)$.) Given a choice of invariant bilinear form and an integral form $V_{\mathbb{Z}}$ of a vertex operator algebra $V$, the contragredient module $V_{\mathbb{Z}}^{\prime}$ may be identified with another lattice spanning $V$ that is invariant under the action of $V_{\mathbb{Z}}$. However, $V_{\mathbb{Z}}^{\prime}$ need not be an integral form of $V$ as a vertex algebra, because it may not be closed under vertex algebra products.

Proposition 6.4. Suppose $V$ is equivalent to $V^{\prime}$ as $V$-module and $V$ has an integral form $V_{\mathbb{Z}}$ preserved by $\frac{L(1)^{n}}{n !}$ for $n \geq 0$; also assume $V_{0}=\mathbb{C} 1$. Identify $V_{\mathbb{Z}}^{\prime}$ with a lattice in $V$ using a non-degenerate invariant form $(\cdot, \cdot)$ such that $(\mathbf{1}, \mathbf{1}) \in \mathbb{Z} \backslash\{0\}$. Then $V_{\mathbb{Z}} \subseteq V_{\mathbb{Z}}^{\prime}$.

Proof. The integral form of $V^{\prime}$ is identified as:

$$
V_{\mathbb{Z}}^{\prime}=\left\{v^{\prime} \in V \mid\left(v^{\prime}, v\right) \in \mathbb{Z} \text { for } v \in V_{\mathbb{Z}}\right\} .
$$

Thus we need to show that if $u, v \in V_{\mathbb{Z}}$, then $(u, v) \in \mathbb{Z}$. We have:

$$
\begin{aligned}
(u, v) & =\operatorname{Res}_{x} x^{-1}(Y(u, x) \mathbf{1}, v) \\
& =\operatorname{Res}_{x} x^{-1}\left(\mathbf{1}, Y\left(e^{x L(1)}\left(-x^{-2}\right)^{L(0)} u, x^{-1}\right) v\right) \\
& =(\mathbf{1}, c \mathbf{1})=c(\mathbf{1}, \mathbf{1})
\end{aligned}
$$

where $c \mathbf{1}=\operatorname{Res}_{x} x^{-1} Y\left(e^{x L(1)}\left(-x^{-2}\right)^{L(0)} u, x^{-1}\right) v$ (since the residue is indeed in $V_{0}$ ). But all coefficients of $Y\left(e^{x L(1)}\left(-x^{-2}\right)^{L(0)} u, x^{-1}\right) v$ are in $V_{\mathbb{Z}}$ because $V_{\mathbb{Z}}$ is closed under vertex operators and invariant under $\frac{L(1)^{n}}{n !}$ for $n \geq 0$. Thus $c \mathbf{1} \in V_{0} \cap V_{\mathbb{Z}}=\mathbb{Z} \mathbf{1}$, by Proposition 2.3 and so $c(\mathbf{1}, \mathbf{1})$ is an integer. 
Remark 6.5. The hypotheses of this proposition are satisfied if $V$ is either a lattice or irreducible affine Lie algebra vertex operator algebra and we choose, for instance, $(\mathbf{1}, \mathbf{1})= \pm 1$.

\section{References}

[B] R. E. Borcherds, Vertex algebras, Kac-Moody algebras, and the Monster, Proc. Natl. Acad. Sci. USA 83 (1986), 3068-3071.

[BR1] R. Borcherds and A. Ryba, Modular moonshine II, Duke Math. J. 83 (1996), no. 2, 435-459.

[BR2] R. Borcherds and A. Ryba, Modular moonshine III, Duke Math. J., 93 (1998), no. $1,129-154$.

[DG] C. Dong and R. Griess, Integral forms in vertex operator algebras which are invariant under finite groups, J. Algebra 365 (2012), 184-198.

[DL] C. Dong and J. Lepowsky, Generalized Vertex Algebras and Relative Vertex Operators, Progress in Math., Vol. 112, Birkhaüser, Boston, 1993.

[FHL] I. Frenkel, Y.-Z. Huang and J. Lepowsky, On axiomatic approaches to vertex operator algebras and modules, Memoirs Amer. Math. Soc. 104, 1993.

[FLM] I. Frenkel, J. Lepowsky and A. Meurman, Vertex Operator Algebras and the Monster, Academic Press, Boston, 1988.

[FZ] I. Frenkel and Y.-C. Zhu, Vertex operator algebras associated to representations of affine and Virasoro algebras, Duke Math. J. 66 (1992), 1-60.

[G] H. Garland, The arithmetic theory of loop algebras, J. Algebra 53 (1978), 490-551.

[GL] R. Griess and C.-H. Lam, Applications of vertex algebra covering procedures to Chevalley groups and modular moonshine, arXiv:1308.2270.

[H] J. Humphreys, Introduction to Lie Algebras and Representation Theory, Graduate Texts in Mathematics, Vol. 9, Springer-Verlag, 1972.

[HLZ] Y.-Z. Huang, J. Lepowsky and L. Zhang, Logarithmic tensor category theory for generalized modules for a conformal vertex algebra, I: Introduction and strongly graded algebras and their generalized modules, arXiv:1012.4193.

[LL] J. Lepowsky and H. Li, Introduction to Vertex Operator Algebras and Their Representations, Progress in Math., Vol. 227, Birkhäuser, Boston, 2003.

[Li] H.-S. Li, Symmetric invariant bilinear forms on vertex operator algebras, J. Pure Applied Algebra 96 (1994), 279-297.

[M] D. Mitzman, Integral bases for affine lie algebras and their universal enveloping algebras, Contemporary Math., Vol. 40, Am. Math. Soc., Providence, 1985. 
[P] S. Prevost, Vertex algebras and integral bases for the enveloping algebras of affine lie algebras, Memoirs Amer. Math. Soc. 466, 1992.

[R] A. Ryba, Modular moonshine?, Moonshine, the Monster, and related topics (South Hadley, MA, 1994), 307-336, Contemp. Math., 193, Amer. Math. Soc., Providence, RI, 1996.

[S] R. Steinberg, Lectures on Chevalley Groups, Yale University mimeographed notes, 1967.

Department of Mathematics, Rutgers University, 110 Frelinghuysen Rd., PiscatAWAY, NJ 08854-8019

E-mail address: rhmcrae@math.rutgers.edu 